\title{
British Thoracic Society Guideline for the initial outpatient management of pulmonary embolism (PE)
}

\author{
Luke S G E Howard, ${ }^{1}$ Steven Barden, ${ }^{2}$ Robin Condliffe, ${ }^{3}$ Vincent Connolly, ${ }^{4}$ \\ Christopher W H Davies, ${ }^{5}$ James Donaldson, ${ }^{6}$ Bernard Everett, ${ }^{7}$ Catherine Free, ${ }_{1}^{8}$ \\ Daniel Horner ${ }_{1}^{9,10}$ Laura Hunter, ${ }_{1}^{11}$ Jasvinder Kaler, ${ }^{12}$ Catherine Nelson-Piercy, ${ }_{1}^{13}$ \\ Emma O'Dowd, ${ }^{14}$ Raj Patel, ${ }^{15}$ Wendy Preston, ${ }^{16}$ Karen Sheares, ${ }^{17}$ Campbell Tait ${ }^{18}$
}

\begin{abstract}
${ }^{1}$ National Pulmonary Hypertension Service, Hammersmith Hospital, Imperial College Healthcare NHS Trust, London, UK ${ }^{2}$ Royal Sussex County Hospital, Brighton, UK

${ }^{3}$ Royal Hallamshire Hospital, Sheffield, UK

${ }^{4}$ South Tees Hospitals NHS Trust, Middlesbrough, UK

${ }^{5}$ Department of Respiratory

Medicine, Royal Berkshire

Hospital, Reading, UK

${ }^{6}$ Derby Teaching Hospitals NHS

Foundation Trust,

${ }^{7}$ Chesterfield, UK

${ }^{8}$ Department of Respiratory

Medicine, George Eliot Hospital,

Nuneaton, UK

${ }^{9}$ Emergency Department, Salford

Royal NHS Foundation Trust,

Salford, UK

${ }^{10}$ The Royal College of

Emergency Medicine, London, UK

${ }^{11}$ St Thomas' Hospital, London, UK

${ }^{12}$ Cardiovascular Department,

Guy's and St Thomas' NHS

Foundation Trust, London, UK

${ }^{13}$ Women's health academic centre, Guy's and Saint Thomas' NHS Foundation Trust, London, UK

${ }^{14}$ Department of Respiratory Medicine, Nottingham City Hospital, Nottingham, UK ${ }^{15}$ King's College Hospital NHS Foundation Trust, London, UK ${ }^{16}$ George Eliot Hospital NHS

Trust, Nuneaton, UK

${ }^{17}$ Royal Papworth Hospital NHS Foundation Trust, Cambridge, UK

${ }^{18}$ Haematology, Glasgow Royal Infirmary, Glasgow, UK
\end{abstract}

\section{Correspondence to}

Dr Luke S G E Howard, National Pulmonary Hypertension Service, Hammersmith Hospital, Imperial College Healthcare NHS Trust, London, W12 OHS, UK;

l.howard@imperial.ac.uk

\section{Check for updates}

To cite: Howard LSGE,

Barden $\mathrm{S}$, Condliffe $\mathrm{R}$, et al.

Thorax Epub ahead of print:

[please include Day Month

Year]. doi:10.1136/

thoraxinl-2018-211539
SUMMARY OF RECOMMENDATIONS

Outcomes of outpatient care for low-risk

pulmonary embolism (PE)

Recommendations

- Patients with PE should be assessed for suitability for management as outpatients (OPs). Grade B

- Patients assessed as low risk and suitable for OP management should be offered treatment in an OP setting where a robust pathway exists for follow-up and monitoring. Grade B

\section{Inclusion and exclusion criteria for $\mathrm{OP}$} management or early discharge

Recommendations

- Patients with confirmed PE should be risk-stratified using a validated clinical risk score. Grade B

- Patients in Pulmonary Embolism Severity Index (PESI) class I/II, simplified version of the PESI (sPESI) 0 or meeting the Hestia criteria should be considered for OP management of PE. Grade B

- Where PESI or sPESI is used and indicates a low risk, a set of exclusion criteria should be applied to patients being considered for outpatient management of confirmed PE. Grade B Exclusion criteria include:

- Haemodynamic instability (HR $>110 \mathrm{bpm}$; systolic blood pressure $(\mathrm{SBP})<100 \mathrm{~mm}$ $\mathrm{Hg}$; requirement for inotropes and critical care; requirement for thrombolysis or embolectomy).

- Oxygen saturations $<90 \%$ on air.

- Active bleeding or risk of major bleeding (eg. recent gastrointestinal (GI) bleed or surgery, previous intracranial bleeding, uncontrolled hypertension).

- On full-dose anticoagulation at the time of the PE.

- Severe pain (eg. requiring opiates).

- Other medical comorbidities requiring hospital admission.

- Chronic kidney disease (CKD) stages 4 or 5 (estimated glomerular filtration rate (eGFR) $<30 \mathrm{~mL} / \mathrm{min}$ ) or severe liver disease.

- Heparin-induced thrombocytopenia (HIT) within the last year and where there is no alternative to repeating heparin treatment.

- Social reasons which may include inability to return home, inadequate care at home, lack of telephone communication, concerns over compliance, etc.
- No specific assessment of bleeding risk is required in patients who are deemed low risk by PESI/sPESI/Hestia criteria. Grade B

- Measurement of right ventricular (RV):left ventricular (LV) ratio on CT or assessment of RV function on echocardiography is not obligatory for the identification of low-risk patients for OP management. Grade C

- Where RV dilatation has been identified on CT scanning or echocardiography in patients who are suitable for OP management, consider measuring laboratory cardiac biomarkers (B-type natriuretic peptide (BNP), N-terminal pro B-type natriuretic peptide (NT-proBNP), high-sensitivity troponin I or $\mathrm{T}$ (hsTnI or hsTnT)). Normal values may be used to identify low-risk patients; elevated biomarkers in this context should prompt inpatient (IP) admission for observation. Grade C

\section{Good practice points}

- In the context of low-risk PE and an incidental finding of elevated troponin, senior review is required and alternative causes for troponin release should be considered.

\section{Management of patients with suspected PE, where a diagnosis has yet to be confirmed Recommendation}

- Patients with suspected PE should, where reasonably practical, undergo investigation on the same day of presentation to exclude a diagnosis of PE. An alternative strategy of anticoagulation followed by OP imaging within 24 hours may be considered in patients with suspected PE, who have been deemed low risk and eligible for OP care as per confirmed PE. Robust systems should be in place to facilitate next day investigation and review. Grade D

Treatment of suspected/confirmed PE in the OP setting

Recommendations

- Patients with confirmed PE being treated in the OP setting should be offered treatment with either low molecular weight heparin (LMWH) and dabigatran, LMWH and edoxaban or a single-drug regimen (apixaban or rivaroxaban). Grade A

- Patients with suspected PE being treated in the OP setting may be treated with apixaban or 
rivaroxaban pending diagnosis as an alternative to LMWH. Grade D

\section{Good practice point}

$\checkmark$ Using a single direct oral anticoagulant (DOAC) in a pathway is preferred to minimise potential confusion over dosing and administration.

\section{Assessing patients transitioning from inpatient care to early discharge/OP care \\ Recommendation}

- Patients who have been admitted with an intermediate risk PE (PESI class III) can be considered for early discharge (ED) when they meet the criteria for low risk (PESI class I/II or sPESI score 0 ). Grade C

\section{Good practice points}

$\checkmark$ Those with PESI-48 class III or sPESI-48 score of $>0$ are considered to be at higher risk of adverse outcome and senior review is necessary prior to discharge; PESI and sPESI may remain elevated due to non-reversible factors (eg, cancer, age) which should be taken into consideration when using clinical judgement.

$\checkmark$ Consideration should be given to repeating assessment of RV function with echocardiography or biomarkers in those admitted with RV dysfunction or biomarker elevation at baseline.

\section{Level of seniority of review}

Good practice points

$\checkmark \quad$ Patients with confirmed or suspected PE should be reviewed by a consultant prior to discharge on an OP PE pathway. If no consultant is available, then patients may be reviewed by a senior trainee (ST3 or above; ST4 in the case of Emergency Medicine) by a staff grade or similar substantive career grade doctor, advanced nurse practitioner or clinical nurse specialist designated to undertake this role within the department with consultant advice available.

$\checkmark \quad$ If patients are on an OP pathway for suspected PE and being considered for discharge and scanning the following day, a local protocol should be in place to guide a full cardiorespiratory assessment to exclude other causes for symptoms (including full history, examination, ECG and chest radiograph), including risk assessment

\section{Follow up of patients specific to those managed in the OP setting \\ Recommendations}

- Patients with confirmed PE who are eligible for OP care should be provided with verbal and written information on the signs and symptoms of recurrence, major bleeding and additional complications. Individual centres should also provide an appropriate point of contact in the event of complications or concerns, both in and out of hours. Grade B

- Patients should have a formal review (telephone/face to face) at least once during the first week after discharge to ensure therapeutic compliance along with the absence of complications. Grade B

- Hospitals should have local protocols and pathways in place for follow-up of all patients with PE, whether treated as an IP or OP. This should include assessment of ongoing symptoms (with further directed investigation as appropriate) and consideration of optimal duration/modality of anticoagulation. Grade D

\section{Good practice points}

$\checkmark$ Consider initial assessment of provoking risk factors for the index PE at an early stage, for example, immobility, surgery, cancer, intercurrent illness, etc, since this will determine duration of anticoagulation. Screening policies for malignancy are out of scope for this guideline, but when screening investigations are performed, a mechanism should be in place to review results within a prompt time frame.

$\checkmark$ Follow-up of PE should be performed by clinicians with a special interest in venous thromboembolism (VTE).

\section{Management of PE in the OP setting in specific circumstances}

Pregnancy/puerperium

Good practice points

$\checkmark$ All pregnant and postpartum women presenting with suspected PE or confirmed PE should be reviewed by a consultant and discussed with maternity services prior to discharge.

$\checkmark$ OP care pathways may be considered for suspected or confirmed PE in pregnancy and/or the postpartum period.

$\checkmark$ Clinical risk scores derived for non-pregnant patients, such as PESI/sPESI, should not be used in pregnant women.

$\checkmark$ DOACs or vitamin K antagonists (VKAs) should not be used in pregnant patients with suspected or proven PE.

\section{Cancer}

Recommendation

$\checkmark$ The Hestia criteria may be used to evaluate patients with active cancer for suitability for OP management of PE. Grade D

\section{Good practice points}

$\checkmark$ Patients with active cancer should be reviewed by a consultant prior to discharge given the higher risk of 30-day mortality.

$\checkmark$ Patients with incidental PE should be managed in the same way with respect to OP management as those with symptomatic PE.

\section{Intravenous drug use}

Good practice point

$\checkmark$ Intravenous drug abusers with suspected PE should be admitted for further investigation and management.

\section{Patient information and support needs \\ Recommendation}

- Written patient information and education should be integral to OP PE pathways. Grade D

\section{Good practice point}

$\checkmark$ Succinct written information should be provided to the patient on discharge, using non-technical language and including telephone numbers/email addresses for advice on dealing with any subsequent changes in the patient's condition. An example, from Sheffield Teaching Hospitals, is included in online supplementary web appendix 3 . The information material produced by the thrombosis charity Thrombosis UK (www.thrombosisuk.org) may also prove helpful. 


\section{INTRODUCTION}

The British Thoracic Society (BTS) Guideline for the initial OP management of PE provides guidance on how to risk-stratify patients with suspected and confirmed PE and subsequently manage them in an OP or ambulatory care setting.

Over the last 10 years there has been an increasing drive to manage many conditions traditionally treated during an IP admission as OPs. This has become widespread practice in managing deep vein thrombosis (DVT) and data are increasing to support this strategy in PE. With the licensing of the DOACs for the treatment of acute PE which do not require a LMWH run-in or International Normalised Ratio (INR) monitoring, OP management of suspected PE has become more straightforward. This is clearly an opportunity to improve patient experience and reduce hospital length of stay (LOS), but it also presents a risk if the wrong patients are identified for OP management.

Many acute hospital trusts have begun OP management of both suspected and confirmed PE, as evidenced by the large number of abstracts presented at conference proceedings, in addition to peer-reviewed publications. However, it is readily apparent from these reports that practice varies, and it is of concern that in some units no validated risk assessment is being undertaken. Given that PE can be fatal, this raises concerns over the safety of some local protocols. This safety concern is further highlighted by the fact that a study of OP management of PE was terminated early by the Drug and Safety Monitoring Board after two deaths in the OP arm (one RV thrombus and one fatal bleed). ${ }^{1}$

The National Institute for Health and Care Excellence (NICE) guidelines on the management of thromboembolic diseases published in 2012 and updated in 2015 provided no recommendations on how to risk-stratify for OP management. ${ }^{2}$ While both the European Society of Cardiology (ESC) and the American College of Chest Physicians have also published guidance on the management of acute PE which both support OP management, neither provides detailed practical recommendations on risk stratification. ${ }^{3-5}$

Finally, the advent of the DOACs facilitates early discharge from hospital since patients no longer need to be kept in hospital until they have reached a therapeutic INR. This raises the question of how to identify moderate-risk patients appropriate for early discharge.

In conclusion, there is a need for a standardised approach to identify low-risk patients for OP management and also to aid clinicians in deciding when moderate-risk patients can be discharged early. The aim of this guideline is to standardise the approach to the initial OP management of PE (up to 3 weeks post diagnosis) and to reduce the risk to patients and hospital trusts, while ensuring best value to the National Health Service.

\section{Target audience for the guideline}

This guideline is aimed at clinicians, in particular physicians, clinical nurse specialists and advanced nurse practitioners, at all levels of seniority delivering emergency, acute, ambulatory and IP care who are involved in the management of acute PE. The main specialties referring to the guidance are likely to be acute, emergency, general (internal) and respiratory medicine. It is also designed to inform general practitioners involved in the delivery of ambulatory care or thrombosis pathways. This document may also be used by healthcare commissioners and hospital management to ensure appropriate staffing and resourcing of ambulatory care facilities to integrate PE management into ambulatory care pathways.

\section{Scope of the guideline}

The guideline covers:

- Adults ( $\geq 16$ years) with suspected and confirmed acute PE.

- Haemodynamically stable PE.

- Use of DOACs in relation to suspected PE and OP management.

- Risk stratification for identifying patients suitable for OP management or early discharge

- Special subgroups of patients (pregnant patients, those with cancer and intravenous drug abusers).

The guideline does not cover:

- The diagnostic algorithm for PE.

- Evidence for cancer screening.

- Detailed comparisons of anticoagulant drugs.

- Who should be treated and not treated, specifically how to manage subsegmental PE.

- Duration of anticoagulation and thrombophilia investigations.

\section{METHODOLOGY}

This guideline is based on the best available evidence. The methodology used to write the guideline adheres strictly to the criteria as set by the AGREE II collaboration, which is available online at http://www.agreetrust.org/resource-centre/agreeii/. The BTS Standards of Care Committee (SOCC) guideline production manual is available at http://www.brit-thoracic.org. uk/guidelines-and-quality-standards/.

\section{Clinical questions and literature search}

Clinical questions were structured in the Population, Intervention, Control, Outcome format (see online supplementary web appendix 1), to define the scope of the guideline and inform the literature search.

Systematic electronic database searches were conducted in order to identify potentially relevant studies for inclusion in the guideline. For each topic area the following databases were searched: Ovid MEDLINE (including MEDLINE In Process), Ovid EMBASE and the Cochrane Library (including the Cochrane Database of Systematic Reviews and the Database of Abstracts of Reviews of Effects) from 1980.

The searches were first run in July 2014 and updated in October 2015 (see online supplementary web appendix 1 for search strategy). Searches included a combination of indexed terms and free text terms and were limited to English-language publications only. The initial search identified 2385 potential abstracts and the second search 432 other abstracts.

\section{Appraisal of literature}

Appraisal was performed to be compliant with the AGREE II instrument. Two individuals (LH, RP) read the title and abstract of each article retrieved by the literature searches and decided whether the paper was definitely relevant, possibly relevant or not relevant to the project. Criteria formulated for categorising the abstracts into these three groups were:

- Whether the study addressed the clinical question.

- Whether the appropriate study type was used to produce the best evidence to answer the clinical question.

- Review articles were excluded.

- Abstract was in English. 


\begin{tabular}{|c|c|}
\hline Grade & Evidence \\
\hline $1++$ & $\begin{array}{l}\text { High-quality meta-analyses, systematic reviews of RCTs or RCTs with a } \\
\text { very low risk of bias }\end{array}$ \\
\hline $1+$ & $\begin{array}{l}\text { Well-conducted meta-analyses, systematic reviews of RCTs or RCTs with a } \\
\text { low risk of bias }\end{array}$ \\
\hline $1-$ & Meta-analyses, systematic reviews of RCTs or RCTs with a high risk of bias \\
\hline $2++$ & $\begin{array}{l}\text { High-quality systematic reviews of case-control or cohort studies or high- } \\
\text { quality case-control or cohort studies with a very low risk of confounding, } \\
\text { bias or chance and a high probability that the relationship is causal }\end{array}$ \\
\hline $2+$ & $\begin{array}{l}\text { Well-conducted case-control or cohort studies with a low risk of } \\
\text { confounding, bias or chance and a moderate probability that the } \\
\text { relationship is causal }\end{array}$ \\
\hline $2-$ & $\begin{array}{l}\text { Case-control or cohort studies with a high risk of confounding, bias or } \\
\text { chance and a significant risk that the relationship is not causal }\end{array}$ \\
\hline 3 & Non-analytic studies, for example, case reports, case series \\
\hline 4 & Expert opinion \\
\hline
\end{tabular}

Abstracts were not rejected on the basis of the journal of publication, the country in which the research was performed or published or the date of publication.

The screened abstracts were allocated to the relevant section(s) of the guideline and two group members allocated to each of those sections. The full paper was obtained for all relevant or possibly relevant abstracts.

The first screening process identified 153 of the initial 2385 reference abstracts to be definitely or possibly relevant to the guideline. Two guideline reviewers per section independently reviewed the abstracts to identify papers to be appraised for the guideline. The two reviewers for each section then independently appraised each paper assigned to them using the Scottish Intercollegiate Guidelines Network (SIGN) critical appraisal checklists. The reliability of the evidence in each individual study was graded using the SIGN critical appraisal checklists and is shown in the evidence tables $(++,+$ or -$)$. The body of evidence for each recommendation was summarised into evidence statements and graded using the SIGN grading system (see table 1).

Disagreements were resolved by discussion with the section partner. The second literature search in October 2015 yielded 432 abstracts. Of these, 13 were identified as definitely or possibly relevant to the guideline. However, all of the pertinent ones from this search had been identified by the Guideline Development Group (GDG) in the meantime and already incorporated.

\section{Considered judgement and grading of evidence}

The GDG used the evidence tables to judge the body of evidence and grade recommendations for this guideline. The evidence tables are available in online supplementary web appendix 2. Where evidence was lacking to answer the formulated clinical questions, expert opinions were obtained through consensus. The following were considered in the grading of the recommendations:

- The available volume of the body of evidence.

- How applicable the obtained evidence was in making recommendations for the defined target audience of this guideline.

- Whether the evidence was generalisable to the target population for the guideline.

- Whether there was a clear consistency in the evidence obtained to support recommendations.

\begin{tabular}{|c|c|}
\hline Grade & Type of evidence \\
\hline \multirow[t]{2}{*}{ A } & $\begin{array}{l}\text { At least one meta-analysis, systematic review or RCT rated as } 1++ \text { and } \\
\text { directly applicable to the target population or }\end{array}$ \\
\hline & $\begin{array}{l}\text { A systematic review of RCTs or a body of evidence consisting principally } \\
\text { of studies rated as } 1+\text { directly applicable to the target population and } \\
\text { demonstrating overall consistency of results }\end{array}$ \\
\hline \multirow[t]{2}{*}{ B } & $\begin{array}{l}\text { A body of evidence including studies rated as } 2++ \text { directly applicable to the } \\
\text { target population and demonstrating overall consistency of results or }\end{array}$ \\
\hline & Extrapolated evidence from studies rated as $1++$ or $1+$ \\
\hline \multirow[t]{2}{*}{ C } & $\begin{array}{l}\text { A body of evidence including studies rated as } 2+\text { directly applicable to the } \\
\text { target population and demonstrating overall consistency of results or }\end{array}$ \\
\hline & Extrapolated evidence from studies rated as $2++$ \\
\hline \multirow[t]{2}{*}{ D } & Evidence level 3 or 4 or \\
\hline & Extrapolated evidence from studies rated as $2+$ \\
\hline$\checkmark$ & $\begin{array}{l}\text { Important practical points for which there is no research evidence, nor is } \\
\text { there likely to be any research evidence } \\
\text { The guideline committee wishes to emphasise these as good practice points. }\end{array}$ \\
\hline
\end{tabular}

- What the implications of recommendations would be on clinical practice in terms of resources and skilled expertise.

- Cost-effectiveness was not reviewed in detail as in-depth economic analysis of recommendations falls beyond the scope of this guideline.

Recommendations were graded from A to D, as indicated by the strength of the evidence, as in table 2. In line with SIGN guidance, evidence appraised as 'minus' was considered in context but in the absence of other supporting evidence appraised as 'plus', it was discussed among the GDG regarding that point and any recommendation hence made was Grade D. Important practical points currently lacking any research evidence and assessed as unlikely to have research evidence in the future were highlighted as 'good practice points' (GPP).

\section{Drafting the guideline}

The GDG corresponded regularly by email, and meetings of the full group were held in April and October 2014, March, May and October 2015, and January 2016 as well as a number of teleconferences. The BTS SOCC reviewed the draft guideline in March 2016. The draft guideline was made available online in January/February 2017 for public consultation and circulated to all the relevant stakeholders. The BTS SOCC re-reviewed the revised draft guideline in June 2017 and final SOCC approval was granted in December 2017.

This BTS Guideline will be reviewed within 5 years of publication.

\section{Representation and stakeholder organisations}

Dr Vincent Connolly represented the Society for Acute Medicine; Dr Chris Davies represented the Royal College of Physicians, London; Dr Daniel Horner and Dr Laura Hunter represented the Royal College of Emergency Medicine; Ms Wendy Preston represented the Association of Respiratory Nurse Specialists; Dr Campbell Tait represented the British Committee for Standards in Haematology.

The following organisations were consulted during the public consultation period:

- Association of Respiratory Nurse Specialists

- Association of Chartered Physiotherapists in Respiratory Care 
- British Cardiovascular Society

- British Committee for Standards in Haematology (The Guideline was peer-reviewed by the British Society for Haematology)

- Primary Care Respiratory Society

- Royal College of Emergency Medicine

- Royal College of Obstetricians and Gynaecologists

- Royal College of Physicians of Edinburgh

- Royal College of Physicians, London

- Royal College of Radiology

- Society for Acute Medicine

- British Lung Foundation

- Thrombosis UK

\section{OUTCOMES OF OP CARE FOR LOW-RISK PE}

Suspected PE is a common cause of presentation to hospital and ranges in severity from small emboli with few or no symptoms through to massive, life-threatening PE. Over the last decade there has been an attempt to characterise a group of low-risk patients who may be able to receive treatment for their PE outside of a traditional IP setting. Studies have sought to identify the proportion of patients who may be considered low risk. The figures range from $37 \%$ to $44 \%$ of patients with confirmed PE who may be considered suitable for home treatment. ${ }^{67}$ In a UK population where the overall incidence of $\mathrm{PE}$ is considered to be $60-70$ cases/ 100000 population, there will be a considerable number of patients who are suitable for OP therapy, even at the lower ranges of reported suitability.

\section{Evidence review}

\section{Outcomes associated with OP management of PE}

There are two randomised controlled trials (RCTs), two meta-analyses and several cohort studies that have explored the outcomes associated with OP management of PE. Comparison of the studies is complicated by the differences in selection criteria and, in some cases, a lack of clarity about what constituted OP or home management of PE.

In the largest RCT, Aujesky et $a l^{8}$ randomised 344 low-risk patients (PESI categories I and II) with confirmed PE, without predefined exclusion criteria based on clinical and social factors, to either IP treatment $(n=172)$ or OP treatment $(n=172)$ with LMWH and VKA as the method of anticoagulation. The trial was designed as a non-inferiority study and the sample size was calculated with $80 \%$ power to detect non-inferiority at a margin of $4 \%$ with a one-sided $\alpha$ of 0.05 , based on an estimated recurrence rate of VTE of $0.9 \%$. A non-inferiority margin of $4 \%$ would test that the true recurrence rates in the OP arm would not exceed the IP arm by $>4 \%$. The study took place in 19 emergency departments in Switzerland, France, Belgium and the USA.

The investigators showed non-inferior outcomes for recurrent VTE at 14 and 90 days with no recurrences in either group at 14 days and one recurrence $(0.6 \%)$ at 90 days in the OP group (95\% upper confidence limit 2.7\%; $\mathrm{p}=0.011$ ). At 14 days, major bleeding outcomes were non-inferior in the OP group with two intramuscular bleeds compared with none in the IP group (95\% upper confidence limit 3.6\%; $\mathrm{p}=0.031$ ). By 90 days, since one patient in the OP group had developed abnormal uterine bleeding, the non-inferiority threshold was exceeded (95\% upper confidence limit $4.5 \% ; \mathrm{p}=0.086$ ). There was no difference in mortality (one death in each arm). OP treatment required a longer duration of LMWH (mean of 11.5 days vs 8.9 days) before therapeutic INR levels were achieved. Patients were well-matched between the two groups.
In the other RCT by Otero et al, ${ }^{1} 132$ low-risk patients with acute symptomatic PE were randomised to early discharge $(n=72)$ or standard hospitalisation $(n=60)$. The statistical methods and analysis were similar to the above study, assuming a $1 \%$ early complication rate (deaths, thromboembolic recurrences and bleeding), although the sample size was calculated with $80 \%$ power and a two-sided $\alpha$ of 0.05 ; the margin for non-inferiority was not specified. Early discharge was defined as after 3 days of admission if a transthoracic echo had excluded right heart strain or after 5 days of admission if an echocardiogram could not be performed. They used a non-validated risk score developed in 2007 by Uresandi et al, ${ }^{9}$ comprising a number of clinical and social exclusion criteria, to identify a low-risk group; however, this only identified $13 \%$ of those who were diagnosed with PE as suitable for home treatment, so was overly selective. The trial found no difference in the rates of recurrent VTE $(2.8 \%$ early discharge vs $3.3 \%$ hospitalised) or significant bleeding (1.4\% vs $1.6 \%$ ) between the groups, but was terminated early due to an unexpectedly high death rate. Overall mortality was 4.2\% (95\% CI $0.5 \%$ to $8.9 \%)$ in the early discharge group and $8.3 \%(95 \%$ CI $1.1 \%$ to $15 \%$ ) in the standard hospitalisation group. Shortterm mortality was $2.8 \%$ (95\% CI $0.8 \%$ to $9.6 \%$ ) in the early discharge group compared with $0 \%$ in the standard hospitalisation group.

There have been two meta-analyses which have attempted to combine the findings from these RCTs and other cohort studies, although as expected largely based on the same studies. ${ }^{10} 11$ There were significant challenges as the studies had differing methods for categorising a low-risk population and defining OP treatment or early discharge from hospital.

Both meta-analyses by Zondag et $a l^{10}$ and Piran $e t ~ a l^{11}$ found that major bleeding events and recurrent VTE rates were low. The pooled rates for major bleeding in those treated as an OP were $0.97 \%$ and $0.81 \%$, respectively. For recurrent VTE the pooled rates in OPs were $1.7 \%$ and $1.47 \%$, respectively. In the meta-analysis by Zondag, in a pooled control group of low-risk patients treated as IPs $(n=383)$ the major bleeding rates and recurrent VTE rates were comparable $(1.0 \%$ and $1.2 \%$, respectively). ${ }^{10}$

In terms of mortality, in the meta-analysis undertaken by Zondag et al, there were 49 reported deaths in those treated as an OP (1.9\%). None of these were due to fatal PE. In the IP group, there were eight deaths $(0.74 \%)$. The pooled incidences did not differ significantly between the groups, and after excluding studies which had over-representation of patients with malignancy ( $>15 \%$ of the study population), the pooled incidence of mortality in the OP group decreased to $0.6 \% .^{5}$ Piran et $a l^{11}$ reported a pooled overall mortality rate of $1.58 \%$ in the OP and early discharge group. The lack of a control group makes comparison difficult. However, these outcomes were comparable to the outcomes of low-risk patients treated in hospital in the RCTs included in this meta-analysis.

\section{Impact on LOS}

The only RCT to report on LOS was that of Aujesky et al. ${ }^{8}$ Ninety-five per cent of patients in the OP arm were managed entirely as OPs, with a mean LOS of 0.5 days from randomisation compared with 3.9 days in the IP arm. ${ }^{8}$ There was no significant difference between the two groups in terms of other healthcare utilisation (hospital readmissions, emergency department visits and OP visits to a doctor's office within 90 days). The only difference between the groups was a higher number of home nursing visits for LMWH administration, as expected, 
in those treated as OPs compared with the IP group (348 vs 105 visits).

There are two other UK studies which have reported data on LOS. Davies et al ${ }^{7}$ studied 157 patients with confirmed PE who were discharged early from hospital on LMWH. The median time to diagnosis was 1.0 (range $0-3 ; 95 \%$ CI 0 to 1 ) days. The length of hospital stay was $1.0(0-3)$ days, distributed as follows: 0-24 hours: 91 (58.0\%); 24-48 hours: 33 (21.0\%); and 48-72 hours: 33 (21.0\%). The total length of OP treatment (bed-days saved) for all patients was 990 days.

A prospective cohort study described the impact of investigating and managing low-risk patients with suspected PE in the OP setting over a 2-year period. Of the 971 patients referred, 871 were treated entirely as OPs $(89.7 \%) .{ }^{12}$ The authors report previous pilot work suggesting this cohort of patients previously had a mean LOS of 1.59 days, resulting in 692 saved bed-days per year.

\section{Patient satisfaction}

Three studies addressed patient satisfaction. There was a lack of consistency in the scoring system used. Those studies which did report patient satisfaction reported favourable scores for OP management.

In the RCT by Aujesky et al, ${ }^{8} 156 / 170$ OPs (92\%) and $158 / 167$ IPs (95\%) were reported to be satisfied or very satisfied with their treatment site. There was no statistical significance between these groups. Of note, $14 \%$ of the patients treated at home expressed that they would have preferred to remain in hospital for longer.

In total, 124 of the 157 patients (79\%) who were discharged early following confirmation of PE completed a satisfaction score in the study by Davies et al. ${ }^{7}$ Of these, $81(65.3 \%)$ gave a score of 10 , indicating that the majority of patients were highly satisfied with OP management. Of the 149 (94.9\%) patients expressing a preference, $144(96.6 \%)$ indicated that they would prefer to receive treatment for a subsequent $\mathrm{PE}$ as an OP.

Agterof et al reported satisfaction and anxiety scores in a cohort of low-risk patients discharged immediately or within 24 hours of PE diagnosis. ${ }^{13}$ The satisfaction score (PSQ-18) was returned on day 10 by $103 / 152$ patients (67.8\%). The mean score for satisfaction with out-of-hospital treatment was 3.8. An anxiety score (HADS-A) was completed at days 0 and 10 by 101 patients $(67.1 \%)$. There was no difference in the mean score on day 0 compared with day 10 (4.29 vs 4.31).

\section{Evidence statement}

In a selected low-risk population treated with LMWH and VKAs, the OP management of acute PE is non-inferior in terms of recurrent VTE events, risk of major bleeding and PE-related death compared with IP care. Evidence level 1+

Management of low-risk PE with LMWH and VKAs in an OP setting results in a reduction in LOS in hospital which may be associated with healthcare-related cost savings. Evidence level $2+$ There is no evidence for improved patient satisfaction with OP versus IP care.

\section{Recommendations}

- Patients with PE should be assessed for suitability for management as OPs. Grade B

- Patients assessed as low risk and suitable for OP management should be offered treatment in an OP setting where a robust pathway exists for follow-up and monitoring. Grade B
Research recommendation

Research is required to enhance the evidence base regarding patient experience and cost effectiveness.

\section{INCLUSION AND EXCLUSION CRITERIA FOR OP MANAGEMENT OR EARLY DISCHARGE}

Accurate risk stratification and identification of those at low risk of morbidity and mortality following a diagnosis of PE can influence management strategies and facilitate OP management or early discharge in those deemed appropriate. This section reviews the evidence surrounding validated risk stratification tools and clinical inclusion and exclusion criteria to OP management following confirmed PE.

\section{Evidence review}

Thirty-four studies were identified that evaluated clinical inclusion or exclusion criteria or derived and/or validated risk prediction tools associated with outcome from PE. The majority of these were retrospective or prospective cohort studies with only two RCTs and three meta-analyses. Only one study was conducted and validated in a UK population; however, the majority were from European or North American populations and the data are, therefore, likely to be representative of patients managed in the UK, accepting that there are some differences in care set-up between countries.

These studies can be grouped into three categories:

1. Studies using clinical risk scores $(n=22)$, including randomised trials of OP versus IP management $(n=2)$.

2. Studies using clinical exclusion criteria $(n=9)$.

3. Meta-analyses $(n=3)$.

\section{Clinical risk scores}

There are several studies both deriving and validating various prediction scores. The most frequently used and most validated scores are the PESI score, Geneva score and sPESI score. A variety of scores have added additional biochemical or radiological investigations to the validated scoring systems to try to refine them further. The studies are summarised in appendices 2 and 3. These scores, although used latterly to select patients for OP management, have originally been derived to predict 30 -day mortality rather than as specific tools to guide decisions regarding selection of patients for OP management.

\section{Pulmonary Embolism Severity Index (PESI)}

Aujesky et $a l^{14}$ derived and validated the PESI using a cohort of 15752 patients with confirmed PE. The score comprises independent predictors of 30-day mortality and is summarised in table 3. Patients with PESI class I (very low risk) and PESI class II (low risk) had a 30 -day mortality of $\leq 1.6 \%$ and $3.6 \%$, respectively. No patients in these classes suffered recurrent VTE or major bleeding during the follow-up period. The score also performed well in internal and external validation with area under the receiver operating characteristic curve (AUC) of 0.77 (95\% CI 0.75 to 0.79 ).

The PESI score has been extensively externally validated. Aujesky et $a l^{15}$ performed an external validation of the PESI using a European dataset. The ability of PESI to predict 90-day mortality and incidence of major bleeding and recurrent VTE within each risk class was assessed. Mortality was 0\% (95\% CI 0 to 5.4 ) and $1.0 \%$ (95\% CI 0 to 5.6) in PESI class I and II, respectively. No patients who were classified as low risk (class I or II) had major bleeding or recurrent VTE at 90 days. The PESI also performed well in validation with AUC of 0.87 (95\% CI 


\begin{tabular}{|c|c|c|c|}
\hline Parameter & Score & Risk class & Total points \\
\hline Demographic features & & I: very low & $\leq 65$ \\
\hline Age & Age in years & \multirow{2}{*}{ II: low } & \multirow{2}{*}{$66-85$} \\
\hline Male sex & +10 & & \\
\hline Comorbid conditions & & III: intermediate & $86-105$ \\
\hline Cancer & +30 & \multirow{2}{*}{ IV: high } & \multirow{2}{*}{$106-125$} \\
\hline Heart failure & +10 & & \\
\hline Chronic lung disease & +10 & V: very high & $\geq 126$ \\
\hline \multicolumn{4}{|l|}{ Clinical findings } \\
\hline Pulse $\geq 110 \mathrm{bpm}$ & +20 & & \\
\hline $\begin{array}{l}\text { Systolic blood pressure < } \\
100 \mathrm{~mm} \mathrm{Hg}\end{array}$ & +30 & & \\
\hline $\mathrm{RR} \geq 30 / \mathrm{min}$ & +20 & & \\
\hline Temperature $<36^{\circ} \mathrm{C}$ & +20 & & \\
\hline Altered mental status* & +60 & & \\
\hline $\begin{array}{l}\text { Arterial blood oxygen } \\
\text { saturation }<90 \% \dagger\end{array}$ & +20 & & \\
\hline
\end{tabular}

${ }^{*}$ Defined as disorientation, lethargy, stupor or coma.

tWith or without the administration of supplemental oxygen.

0.80 to 0.94$)$. Further studies have shown similar findings and on average identify $50 \%$ of patients as being low risk. ${ }^{1617}$

PESI was subsequently used in the randomised trial of OP versus IP management of PESI I/II patients with PE being randomised to receive IP or OP care but in conjunction with other exclusion criteria for suitability for OP management. ${ }^{8}$

\section{Simplified Pulmonary Embolism Severity Index (sPESI)}

sPESI was derived by Jiménez et $a l^{18}$ and externally validated using data from a large international registry (Registro Informatizado de la Enfermedad TromboEmbólica (RIETE)). The score consists of six variables which are listed in table 4. A score of zero is classified as low risk. The original PESI was also applied to the sPESI derivation cohort to provide a comparison with a well-validated score. The sPESI was shown to be non-inferior to the PESI in predicting 30-day mortality. sPESI classified $30.7 \%$ of patients as low risk in the derivation cohort and $36.2 \%$ of patients in the validation cohort as low risk compared with $36.3 \%$ using the PESI. Thirty-day mortality in these low-risk groups were $1.0 \%$ (95\% CI 0.69 to 0.80 ) and $1.1 \%$ (95\% CI $0.7 \%$ to $1.5 \%$ ), respectively. There was a low incidence of major bleeding and recurrent VTE in those classified as low risk by sPESI of $\leq 1.5 \%$ at 30 days in both the derivation and validation groups.

\begin{tabular}{llll}
\hline Table 4 & Simplified Pulmonary Embolism Severity Index & \\
\hline Parameter & Score & Risk class & Total points \\
\hline Age $>80$ years & 1 & Low & 0 \\
Cancer $^{*}$ & 1 & High & $\geq 1$ \\
Chronic cardiopulmonary disease & 1 & & \\
Pulse $\geq 110$ bpm & 1 & & \\
Systolic blood pressure $<100 \mathrm{~mm} \mathrm{Hg}$ & 1 & & \\
Arterial blood oxygen saturation $<90 \% \dagger$ & 1 & & \\
\hline
\end{tabular}

*Defined as active cancer (diagnosed within last 12 months or undergoing treatment, personal communication from Prof David Jimenez).

tWith or without the administration of supplemental oxygen.

\section{Geneva score}

The Geneva score, derived in 2000, uses a combination of clinical and subjective criteria with weighting towards cancer and heart failure. The score also requires the use of leg ultrasound (USS) to identify DVT and may not be readily applicable to a UK-based system of investigation for PE.

The original derivation was performed by Wicki et $a l^{19}$ and identified six features as predictive of mortality, recurrent VTE or bleeding at 90 days. The Geneva score is summarised in table 5. Of 296 patients, $67.2 \%$ were deemed to be low risk using the Geneva score, with 2.2\% 90-day mortality.

The Geneva score was validated by Nendaz et $a l^{20}$ In their cohort, the Geneva score identified $79.9 \%$ of patients as low risk (score $\leq 2$ ), with 5\% (95\% CI 2.6 to 9.6\%) experiencing an adverse event within the first 90 days (defined as death, major bleed or recurrent VTE). The model appeared to show good discriminative ability in this population to predict 90 - day adverse events (AUC 0.77 (95\% CI 0.65 to 0.89)).

\begin{tabular}{|c|c|c|c|}
\hline Parameter & Score & Risk class & Total points \\
\hline Cancer & 2 & Low & $\leq 2$ \\
\hline Heart failure & 1 & High & $>2$ \\
\hline Previous DVT & 1 & & \\
\hline $\mathrm{SBP}<100 \mathrm{~mm} \mathrm{Hg}$ & 2 & & \\
\hline $\mathrm{PaO}_{2}<8 \mathrm{kPa}(60 \mathrm{~mm} \mathrm{Hg})$ & 1 & & \\
\hline DVT confirmed on USS & 1 & & \\
\hline
\end{tabular}

AUC, area under the receiver operating characteristic curve; DVT, deep vein thrombosis; SBP, systolic blood pressure; USS, ultrasound; VTE, venous thromboembolism.

\section{Other risk prediction scores}

Various other scores have been derived, ${ }^{21-25}$ some including hybrids of clinical features and biochemical markers such as d-dimer and NT-proBNP 2223 ; however, these suffer from a mixture of lack of validation $^{22-24}$ and/or identification of small numbers of patients as low risk, thus reducing clinical utility. 212425

Most of the scores discussed above evaluate outcomes at 30 days, yet it may be more relevant to consider systems which risk-stratify patients for early adverse events when integrating into OP pathways. Maestre et $a l^{26}$ used an international registry of 18707 patients with PE (RIETE) to derive a new score to examine 10-day composite outcome of recurrent PE, major bleeding or death. This score (RIETE score) was compared with PESI and sPESI. The RIETE score comprised 11 independent predictors, which included some markers of comorbidity, cardiovascular instability and hypoxia. RIETE classified as low risk more patients than PESI but fewer than sPESI (24\%, 17\% and $35 \%$, respectively). Area under the ROC curve was similar for all three scores, although in internal validation the RIETE score did show slightly greater discrimination. The authors conclude that this score requires further external validation.

\section{Studies validating and comparing multiple predictive scores}

Erkens $e a^{27}$ used a Canadian dataset to validate and compare PESI and sPESI. In their cohort, 48.6\% (95\% CI 42.1\% to 55\%) of patients were deemed low risk using the PESI (class I or II) and $33.3 \%$ (95\% CI $27.4 \%$ to $39.6 \%$ ) were identified as low risk using sPESI. The low-risk patients had 0\% 90-day mortality, with no adverse events (bleeding or recurrent VTE) within the first 14 days. 
Jiménez $e t a l^{28}$ aimed to validate and compare the PESI with the Geneva score in a cohort of 599 patients. The end points were mortality, major bleeding and recurrent VTE at 30 days. The PESI classified substantially fewer patients as low risk (class I and II) compared with the Geneva score (36\%; 95\% CI 32\% to $40 \%$ vs $84 \%$; $95 \%$ CI $81 \%$ to $87 \%$ ). There was no significant difference between the low-risk PESI and Geneva groups in terms of bleeding or recurrent VTE; however, there was a marked and significant difference in 30-day mortality between the two groups identified as low risk. PESI class I and II had $0.9 \%$ (95\% CI $0.3 \%$ to $2.2 \%$ ) mortality, with the Geneva low-risk group mortality much higher at $5.6 \%$ (95\% CI 3.6\% to $7.6 \%)$. When the discriminatory power of the scores was assessed by means of ROC curve the PESI showed much better discriminatory ability (AUC $0.76(95 \%$ CI 0.69 to 0.83 ) and $0.61(95 \%$ CI 0.51 to 0.71 ), respectively). This suggests that PESI is better at identifying those patients at low risk of 30-day mortality than the Geneva score.

Kabrhel et al ${ }^{29}$ studied the features which predicted clinical deterioration and need for intervention within the first five days after diagnosis of PE and also 30-day, all-cause mortality. The features which were found to be independent predictors included SBP $<90 \mathrm{~mm} \mathrm{Hg}$, arterial saturations $<90 \%$, coronary artery disease, residual DVT and right heart strain on echocardiography. Active malignancy and heart failure were associated with increased 30-day mortality. The authors also validated PESI, sPESI and Geneva scores for a variety of outcomes, including a composite outcome that included mortality, use of vasopressors, dysrhythmia and need for ventilation among others parameters. The more comparable AUC calculation for 30-day mortality for PESI was 0.84 , sPESI 0.82 and Geneva 0.78 and these scores showed high sensitivity for 30-day mortality. The authors concluded that, in their cohort, $66 \%$ of patients suffered no clinical deterioration and had no need for intervention in the initial five days after diagnosis and would thus be deemed suitable for OP management.

\section{Clinical exclusion criteria}

Several studies have derived a number of clinical criteria for which discharge as an OP is considered inappropriate. These included obvious variables indicating serious or potentially life-threatening PE, such as the need for thrombolysis or the need for inotropes and intensive care unit support. Early studies, before prediction scores, used variables such as hypoxaemia and hypotension, pain requiring analgesia, bleeding or high risk of bleeding and subjective criteria allowing exclusion if required to stay for a medical reason or likely poor compliance or social factors. The 'Hestia' criteria have been the most widely used and are presented in table 6 alongside the only criteria to have been derived from a UK population.

The Hestia criteria are a series of exclusion criteria which were proposed in 2011 by Zondag et al using data from a series of small observational studies looking at clinical selection for OP management in confirmed PE (table 6). ${ }^{30}$ They have been validated in prospective cohort studies ${ }^{30}{ }^{31}$ but not in an RCT whereby patients who meet the criteria are randomised to IP or OP care. The derivation study used these criteria alone to stratify patients into OP (discharge $<24$ hours after diagnosis) or standard care and looked at incidence of recurrent VTE, major bleeding and mortality at 90 days. Fifty-one per cent of patients diagnosed with PE were deemed eligible for initial OP management using the Hestia criteria. Of these, 2\% (95\% CI $0.8 \%$ to $4.3 \%$ ) suffered recurrent VTE, with $1 \%$ (95\% CI $0.2 \%$ to $2.9 \%$ ) all-cause mortality at 3 months and $0.7 \%$ (95\% CI $0.08 \%$ to 2.4\%) experiencing major bleeding. Of note, when the Hestia criteria are not met, this identifies a high-risk group at similar risk of 3 -month mortality $(9.6 \%)$ as sPESI $>0 .^{32}$

Table 6 Clinical exclusion criteria for outpatient PE management

\begin{tabular}{|c|c|c|c|}
\hline \multicolumn{2}{|l|}{ Hestia criteria (Zondag et al) ${ }^{30}$} & \multicolumn{2}{|l|}{ Davies et al ${ }^{7}$} \\
\hline Is the patient haemodynamically unstable?* & Yes/no & Need for hospitalisation for another medical reason & Yes/no \\
\hline Is thrombolysis or embolectomy necessary? & Yes/no & $\begin{array}{l}\text { Additional monitoring required (ECG, oxygen, parenteral } \\
\text { analgesia) }\end{array}$ & Yes/no \\
\hline Active bleeding or high risk of bleeding?† & Yes/no & Likelihood of poor compliance/difficulty with follow-up & Yes/no \\
\hline $\begin{array}{l}\text { More than } 24 \text { hours of oxygen supply to maintain oxygen } \\
\text { saturation }>90 \% \text { ? }\end{array}$ & Yes/no & Previous PE/early recurrence of PE & Yes/no \\
\hline Is PE diagnosed during anticoagulant treatment? & Yes/no & Coexisting major DVT & Yes/no \\
\hline $\begin{array}{l}\text { Severe pain needing intravenous pain medication for }>24 \\
\text { hours? }\end{array}$ & Yes/no & Bleeding disorders, active bleeding & Yes/no \\
\hline $\begin{array}{l}\text { Medical or social reason for treatment in hospital > } 24 \\
\text { hours? }\end{array}$ & Yes/no & Pregnancy & Yes/no \\
\hline Does the patient have a creatinine clearance $<30 \mathrm{~mL} / \mathrm{min}$ ? & Yes/no & Patient preference for hospital stay & Yes/no \\
\hline $\begin{array}{l}\text { Does the patient have severe liver impairment? } \\
\text { (discretion of clinician) }\end{array}$ & Yes/no & & \\
\hline Is the patient pregnant? & Yes/no & & \\
\hline $\begin{array}{l}\text { Does the patient have a documented history of heparin- } \\
\text { induced thrombocytopenia? }\end{array}$ & Yes/no & & \\
\hline
\end{tabular}

Eligible for outpatient treatment-no risk factors.

Ineligible for outpatient treatment-at least one risk factor present.

\footnotetext{
${ }^{*} \mathrm{SBP}<100 \mathrm{~mm} \mathrm{Hg}$ with $\mathrm{HR}>100 \mathrm{bpm}$; condition requiring admission to intensive care unit

$\dagger$ Gastrointestinal bleeding in the preceding 14 days, recent stroke $(<4$ weeks ago), recent operation ( $<2$ weeks ago), bleeding disorder, thrombocytopenia (platelet count $<75 x$ $\left.10^{9} / \mathrm{l}\right)$, uncontrolled hypertension (SBP $>180 \mathrm{~mm} \mathrm{Hg}$ or DBP $>110 \mathrm{~mm} \mathrm{Hg}$ ).

DVT, deep vein thrombosis; DBP, diastolic blood pressure; SBP, systolic blood pressure.
} 


\section{Box 1 Clinical exclusion criteria ${ }^{8}$}

- Oxygen saturation $<90 \%$

- Systolic blood pressure $<100 \mathrm{~mm} \mathrm{Hg}$

- Chest pain needing opiates

- Active bleeding

- High risk of bleeding (stroke within the preceding 10 days, gastrointestinal bleed within the last 14 days or platelet count $\left.<75000 / \mathrm{mm}^{3}\right)$

- Obesity (weight $>150 \mathrm{~kg}$ )

- Heparin-induced thrombocytopenia

- Severe renal failure (creatinine clearance $<30 \mathrm{~mL} / \mathrm{min}$ )

- Therapeutic anticoagulation (International Normalised Ratio $\geq 2.0$ ) at diagnosis

- Barriers to treatment adherence or follow-up

The only tool derived and validated in the UK was developed by Davies $e t \mathrm{al}^{7}$ in 2007. It comprises a series of eight clinical exclusion criteria which are also summarised in table 6 . In the derivation study, $43.6 \%$ of patients were deemed suitable for OP management using these criteria. During the 90-day follow-up, $4.5 \%$ died, with bleeding (both major and minor) occurring in $5 \%$ and recurrent VTE in 3\%. Application of these criteria to the validation cohort seemed to identify a population with low risk of adverse events at 90 days (recurrent VTE 0\% (95\% CI $0.0 \%$ to $2.3 \%$ ); major bleeding $0 \%(95 \%$ CI $0.00 \%$ to $2.3 \%)$ and death $1.9 \%$ (95\% CI $0.4 \%$ to $5.5 \%)$ ).

Many other investigators have used very similar criteria with similar findings, ${ }^{33-35}$ although one study by Elf $e t a l^{6}$ included the extent of perfusion defect on VQ scan, making it impractical for use in the UK presently. These are presented in appendices 2 and 3.

Although the randomised controlled study by Aujesky et $a l^{8}$ used PESI to identify low-risk patients, they also applied bespoke exclusion criteria (Box 1), many of which appear to overlap with criteria in PESI and also bear many similarities with the Hestia criteria.

\section{Studies comparing clinical exclusion criteria with risk scores}

Zondag $\mathrm{et} \mathrm{al}^{36}$ compared the Hestia criteria with sPESI for 30-day mortality by conducting a post-hoc analysis on the original Hestia derivation dataset. Of 468 patients in whom sPESI could be calculated, $53 \%$ were identified as suitable for OP management by Hestia criteria, with $59 \%$ deemed low risk according to sPESI. Although both tools selected slightly different patients as low risk, they had similar outcomes, with 30-day adverse events (major bleeding, recurrent VTE or death) of $2.4 \%$ in the Hestia group and $2.2 \%$ in the sPESI low-risk patients. sPESI performed slightly better than Hestia in testing (AUC 0.756 (95\% CI 0.642 to 0.871 ) vs 0.679 (95\% CI 0.536 to 0.822 ), respectively).

A further post-hoc analysis in 2013 compared the Hestia derivation cohort with ESC criteria (shock, PESI/sPESI, RV impairment on imaging, biomarkers) for predicting 90-day adverse events. ${ }^{37}$ Both the Hestia criteria and European Society of Cardiology (ESC) criteria identified over half of the patients as low risk and potentially suitable for OP management $(55 \%$ and $54 \%$, respectively). Again, although they selected a slightly different patient group as suitable for OP management, their negative predictive value (NPV) for adverse events was very similar (100\% for Hestia and 99\% for ESC score).

Vali et $a l^{12}$ used a modified version of the PESI in combination with a series of clinical exclusion criteria (pulse $<110 \mathrm{bpm}$,
SBP $>100 \mathrm{~mm} \mathrm{Hg}$, oxygen saturations $>92 \%$ on air, respiratory rate $<30 \mathrm{bpm}$, no syncope, normal troponin, no large central clot or right heart strain on CTPA, no compliance or follow-up issues, low risk of bleeding, did not require admission for other comorbid conditions) to identify patients with suspected PE who were suitable for OP management. Using these criteria they managed $72.9 \%$ of patients with confirmed PE as OPs with PE-specific mortality of $0 \%$.

\section{Meta-analyses}

There are three meta-analyses which broadly draw the same conclusions.

Squizzato et al $^{38}$ looked at 33 studies comprising 35518 patients and compared clinical prediction tools for complications following treatment of low-risk PE. The authors excluded any prediction tools which incorporated markers of RV dysfunction which required invasive investigation (eg, systolic pulmonary artery pressure). There was a pooled mortality at 30 days of $1.7 \%$, at 14 days of $0.7 \%$ and at 90 days of $2.2 \%$. Non-fatal recurrent VTE and major bleeding at 14, 30 and 90 days, respectively, were $0.8 \%, 1.8 \%$ and $2.3 \%$. The meta-analysis concluded that clinical prediction tools can be used to safely identify patients with acute PE at low risk of complications.

Zondag et $a l^{10}$ looked at low-risk patients managed as OPs $(<24$ hours; $n=1657)$, early discharge (ED) $(<72$ hours; $n=256)$ or IPs $(n=383)$ and compared outcomes. Results showed a low pooled incidence of recurrent VTE $(1.7 \%, 1.1 \%$ and $1.2 \%$, respectively, OP, ED and IP), low pooled major bleeding $(0.97 \%$, $0.78 \%$ and $1.0 \%$, respectively) and low pooled mortality $(1.9 \%$, $2.3 \%$ and $0.74 \%)$. This suggests that low-risk patients can be managed as safely in the OP setting as following early discharge from the IP setting.

Piran $e a^{11}$ also assessed pooled data on 1258 patients in eight prospective cohorts and RCTs looking at OP treatment and early discharge (1-3 days), showing pooled event rates at 3 months. Overall mortality was $1.58 \%$, recurrent VTE rates $1.47 \%$, fatal PE $0.047 \%$ and major bleeding $0.81 \%$. Clinical and risk stratification models appear effectively to risk-stratify low-risk patients who would be suitable for OP management for acute PE.

\section{ASSESSMENT OF BLEEDING RISK}

Historically patients with confirmed PE are initially treated with anticoagulants in a hospital setting to avoid potential complications including major bleeding. Major bleeding is defined according to the ISTH criteria: (i) fatal bleeding and/or (ii) symptomatic bleeding in a critical area or organ, such as intracranial, intraspinal, intraocular, retroperitoneal, intra-articular or pericardial, or intramuscular with compartment syndrome and/or (iii) bleeding causing a fall in haemoglobin level of $20 \mathrm{~g} / \mathrm{L}$ or more or leading to transfusion of two or more units of whole blood or red cells.

Many studies examining major bleeding rates have assessed these over 3 months or longer, whereas bleeding in the first 7-14 days may be more relevant for risks related to OP PE management. In the RIETE registry of 24395 patients with VTE on anticoagulation, $2.24 \%$ had a major bleed and $0.55 \%$ had a fatal bleed during the first three months of anticoagulation. ${ }^{39}$

\section{Evidence review}

The RIETE investigators have derived and validated a score in patients with documented VTE to predict the risk of major 
bleeding within 3 months of anticoagulant therapy. ${ }^{40}$ On multivariate analysis, age $>75$ years, recent bleeding, cancer, creatinine levels $>1.2 \mathrm{mg} / \mathrm{dL}$, anaemia or PE itself were independently associated with an increased risk of major bleeding. The same registry was used to derive a scoring system for early adverse outcomes (the composite outcome included all-cause mortality, recurrent PE and major bleeding) within 10 days of acute PE. ${ }^{26}$ Within the 18707 cohort, of whom 95.1\% had been managed as IPs, major bleeding was reported in $203(1.09 \%)$. A low RIETE score, defined as having none of the nine characteristics associated with an increased risk of the composite outcome, was associated with a low bleed rate $(0.2 \%)$ which was lower than patients with a low sPESI $(0.48 \%)$, but no different from those with a very low PESI $(0.31 \%)$. Other scoring systems, for example, HASBLED, have been largely developed and validated in AF cohorts. In a retrospective study of 223 patients with PE and 314 with DVT who were commenced on VKA between 2006 and 2007 in three hospitals in the Netherlands, the HASBLED score was used to estimate the risk of major bleeding within 180 days. $^{41}$ Cumulative incidences of major bleeds were $1.3 \%$ (95\% CI 0.1 to 2.5 ) in the non-high risk group (HASBLED <3) and $9.6 \%$ (95\% CI 2.2 to 17.0 ) in the high-risk group (HASBLED $\geq 3$ ) ( $\mathrm{p}<0.0001$ by log-rank test), with an HR of 8.7 (95\% CI 2.7 to 28.4). Abnormal renal function (HR 10.8, 95\% CI 1.9 to 61.7) and a history of bleeding events (HR 10.4, 95\% CI 2.5 to 42.5 ) were independent predictors of major bleeds during follow-up. However, 388 of the 537 patients had missing variable(s) and the median time to a major bleed was 61 days (min 6, max 148 days).

Klok et $a l^{42}$ externally validated and compared the predictive value of the HEMORR2HAGES, HASBLED and ATRIA scores to the Kuijer and RIETE scores for the occurrence of major bleeding complications over a 30-day period in 448 consecutive patients with PE treated with a VKA. Most bleeding events $(16 / 20)$ occurred in the first seven days after treatment initiation, with four bleeding complications between days 8 and 30. The predictive power of all five scores for bleeding was poor (c-statistics $0.57-0.64$ ), both for the three-level and two-level score outcomes. No individual score was found to be superior. The HASBLED score had a good c-statistic for bleeding occurring after the first week of treatment $(0.75,95 \%$ CI 0.47 to 1.0$)$. There are no studies deriving or validating an early bleeding risk score specifically for OP PE management.

In the open-label, non-inferiority trial discussed in the previous section by Aujesky et $a l^{8} 344$ patients with acute PE and PESI risk class I or II were randomised to IP treatment or discharge from hospital within 24 hours after randomisation. Active bleeding and high risk of bleeding, including severe renal impairment, were important and relevant exclusion criteria. Also, 2 (1.2\%) of 171 OPs and none of the IPs had major bleeding within 14 days (95\% UCL 3.6\%; $\mathrm{p}=0.31$ ), suggesting in selected low-risk patients OP care was as safe as IP treatment.

In a multicentre prospective randomised trial of home treatment $(n=72$; discharged at day 3 when transthoracic echocardiogram (TTE) excluded RV dysfunction or day 5) versus hospitalised therapy $(n=60)$, the rates of clinically relevant bleeding were $5.5 \%$ in the early discharge group and $5 \%$ in the IP group (relative risk (RR) 1.11 , 95\% CI 0.26 to 4.77 ; $\mathrm{p}=0.60){ }^{1}$ The incidence of major bleeding was $1.4 \%$ in the early discharge group and $1.6 \%$ in the IP group for a RR of 0.83 (95\% CI 0.05 to 13.05 ). The incidence of minor bleeding was $4.2 \%$ in the early discharge group and $3.3 \%$ in the IPs with a RR of 1.25 (95\% CI 0.22 to 7.24 ). As the short-term mortality was $2.8 \%$ (95\% CI $0.8 \%$ to $9.6 \%$ ) in the early discharge group compared with $0 \%$ in the standard hospitalisation group, the study was suspended. One death was due to GI haemorrhage at day 5. The criteria to select patients for this study were not validated.

A meta-analysis of RCTs and prospective or retrospective cohort studies looked at low-risk patients assessed by a variety of risk factors (many used a variation of Hestia criteria) and their pooled incidence of major bleeding. ${ }^{10}$ The pooled incidence of major bleeding was $0.97 \%$ (95\% CI $0.58 \%$ to $1.6 \%)$ in OPs, $0.78 \%$ (95\% CI $0.16 \%$ to $3.73 \%$ ) in early discharge patients (<72 hours) and $1 \%(95 \%$ CI $0.39 \%$ to $2.8 \%)$ in IPs. Piran et al performed a systematic review and pooled meta-analysis of three RCTs and eight prospective cohort studies examining OP versus early discharge (1-3 days). ${ }^{11}$ Also, 5 of 11 studies used risk stratification models (eg. PESI and Uresandi) and the remainder used clinical gestalt. The rates of major bleeding and fatal intracranial haemorrhage were $0.81 \%\left(95 \%\right.$ CI $0.37 \%$ to $\left.1.42 \% ; \mathrm{I}^{2}: 0 \%\right)$ and $0.29 \%(95 \%$ CI $0.06 \%$ to $0.68 \%$; $\left.\mathrm{I}^{2}: 0 \%\right)$, respectively. The pooled rate of major bleeding for patients stratified using clinical gestalt was $0.62 \%$ (95\% CI $0.059 \%$ to $1.79 \%$; I $: 14.7 \%$ ) compared with $0.94 \%$ (95\% CI $0.4 \%$ to $1.8 \%$; $\mathrm{I}^{2}: 25 \%$ ) for patients identified using a risk stratification model. The short-term ( $<14$ days) pooled risk of major bleeding was $0.46 \%$ (95\% CI $0.022 \%$ to $\left.1.46 \% ; \mathrm{I}^{2}: 30 \%\right)$. Independent of the risk stratification method used, the risk of major bleeding was low. None of these studies assessed the bleeding risk in patients treated with non-vitamin-dependent DOACs. The risk of bleeding with these agents is discussed later.

\section{IMAGING, LABORATORY AND OTHER CLINICAL INVESTIGATIONS FOR RISK STRATIFICATION}

Risk stratification using a validated scoring tool, together with clinical exclusion criteria, can identify patients who are suitable for OP management; approximately $1 \%-2 \%$ of this cohort will undergo a complicated clinical course. Additional markers of PE severity may further reduce this risk, although potentially at the cost of reducing the number of patients suitable for OP management with only minimal further risk reduction. Further measurements may also add to time and cost.

\section{Evidence review}

\section{NT-proBNP and BNP}

Agterof $e t a l^{13}$ prospectively studied 351 haemodynamically stable patients with acute PE. One hundred and fifty-two patients (43\%) had both an absence of exclusion criteria and NT-proBNP $<500 \mathrm{pg} / \mathrm{mL}$ and were discharged $<24$ hours following PE diagnosis. No deaths, recurrent VTE or major bleeding up to 3 months were observed. Klok et $a l^{43}$ had earlier performed a meta-analysis of six studies $(n=543)$ of NT-proBNP and seven studies $(n=589)$ of BNP in acute PE. Elevated levels of both BNP and NT-proBNP were associated with worse outcomes (combined 30-day mortality OR 7.6 (3.4-17)). In a more recent study, patients who met the Hestia criteria for OP management of PE were randomised to direct discharge or NT-proBNP testing; those with elevated NT-proBNP were admitted for IP management. ${ }^{31}$ The study confirmed that patients meeting the Hestia criteria had low rates of adverse outcomes and failed to show an additional benefit of NT-proBNP testing, possibly due to low numbers of patients with elevated NT-proBNP levels. 


\section{Standard troponin}

Jimenez $e t a l^{44}$ studied the utility of standard cardiac troponin I (cTnI) in identifying high-risk and low-risk patients in a cohort of 318 patients with non-massive acute PE. Jimenez et $a l^{45}$ subsequently performed a meta-analysis of all available studies of standard cTnI and standard cardiac troponin $\mathrm{T}$ (cTnT). Both studies concluded that standard cardiac troponins did not, on their own, adequately distinguish between normotensive patients at low or high risk of deterioration. Moore et $a l^{46}$ subsequently studied both standard cTnT and PESI in 567 patients with acute PE and observed that low-risk PESI (I/II) had a higher NPV and negative likelihood ratio (NLR) than a negative standard $c \operatorname{TnT}(<0.1 \mathrm{ng} / \mathrm{mL})$. Thirty-four per cent were classified as low risk by PESI alone but the addition of cTnI reduced this proportion to $26 \%$. Furthermore, the addition of cTnT to PESI did not significantly improve the NPV or NLR to predict mortality at 30 days.

\section{High-sensitivity troponin}

The introduction of hsTnI and hsTnT has provided a potentially superior method of identifying patients with extremely low levels of myocardial ischaemia and, hence, increase the ability to detect those at risk of early deterioration. Lankeit et al ${ }^{47}$ performed a prospective multicentre study of 526 normotensive patients and assessed both sPESI and hsTnT. Two patients (1\%) with sPESI 0 and four patients (2\%) with hsTnT $<14 \mathrm{pg} / \mathrm{mL}$ had complicated outcomes compared with zero patients with both sPESI 0 and hsTnT $<14 \mathrm{pg} / \mathrm{mL}$, suggesting that the combination of hsTnT and sPESI may be superior to either parameter alone. Twenty-four per cent of the study population had both an sPESI of 0 and an hsTnT $<14 \mathrm{pg} / \mathrm{mL}$. Ozsu et $a l^{48}$ prospectively assessed sPESI, standard cTnT and hsTnT in 121 normotensive patients and observed that hsTnT was superior to standard cTnT in identifying low-risk and high-risk patients. As the NPV of an sPESI of 0 was $100 \%$ in this cohort, the addition of hSTnT to sPESI did not improve identification of very low-risk patients. Hakemi et al ${ }^{49}$ performed a retrospective, single-centre study of 298 consecutive admissions with PE. No patients with hsTnI $<0.012 \mathrm{ng} / \mathrm{mL}$ died or required thrombolysis or cardiopulmonary resuscitation. Eighty-nine (30\%) patients had both an hsTnI $<0.012 \mathrm{ng} / \mathrm{mL}$ and a PESI of I or II; these patients were at lower risk of requiring admission to the intensive care unit or inferior vena cava filter insertion. However, the exact NPV is not clear.

\section{Echocardiography}

No studies have specifically assessed the additional information supplied by echocardiography in addition to clinical risk score. Jimenez et $a l^{50}$ studied 214 haemodynamically stable patients. Although the NPV of a normal echocardiographic examination for 30-day mortality was high (98\%), the positive predictive value was only $5 \%$. Furthermore, the NLR was 0.71 , leading the authors to conclude that echocardiography added little value to clinical criteria in identifying low-risk or high-risk patients. A meta-analysis by Coutance et $a l^{51}$ identified eight studies assessing echocardiography $(n=1249)$ and also calculated a suboptimal NLR of 0.62 , suggesting that echocardiography has limited use in identifying low-risk patients suitable for early discharge.

\section{CT}

Becattini et $a l^{52}$ performed a meta-analysis of 4767 normotensive and hypotensive patients from 27 studies which investigated the ratio of RV to LV diameter on CT. Patients were subsequently grouped as positive or negative for RV dilatation based on CT findings only, typically based on an RV/LV cut-off ratio of 0.9 or 1 . The NPV for 30-day all-cause mortality was 95\% and for PE-related mortality 99\%. The authors therefore suggested that lack of RV dilatation on CT may have utility in identifying patients suitable for OP treatment. However, in a prospective multicentre study of 848 normotensive patients, Jimenez et $a l^{53}$ observed no difference in 30-day all-cause mortality between patients with and without RV dilatation on CT (4.7\% vs $4.3 \%$; $\mathrm{p}=0.93)$. In this study, the two independent prognostic predictors identified were standard cTnI and BNP. Zondag et $a l^{37}$ performed further analysis of the Hestia data and investigated 496 patients with CT data. No adverse events were observed in the 275 patients treated at home on the basis of their Hestia criteria. Thirty-five per cent of this group had RV dilatation on CT, suggesting that the Hestia criteria were superior to CT assessment of RV dilatation in identifying low-risk patients.

\section{Other prognostic markers}

Several other parameters have been identified as potentially useful in identifying patients with PE at higher risk of mortality. However, limited data exist to support routine use of these parameters in helping to identify patients at very low risk. Shopp et al recently performed a meta-analysis of 10 studies of ECG in acute PE, involving 3007 patients. Six ECG parameters were associated with poorer 30-day outcome: heart rate $>100$ beats/ min, S1Q3T3, complete RBBB, inverted T waves in V1-V4, ST elevation in aVR and atrial fibrillation. ${ }^{41}$ The Daniel score (a 21-point scoring system) was significantly lower in those patients without 30-day haemodynamic collapse (mean $2.6 \pm 1.5$ cf. $5.9 \pm 3.9$ ). There was, however, no comparison of the Daniel score with PE clinical risk scores or other biomarkers.

Jimenez et $a l^{54}$ performed bilateral leg compression ultrasonography in 707 consecutive patients diagnosed with acute PE. The presence of DVT was associated with increased all-cause mortality (adjusted HR 1.66 (1.28 to 2.15)). Aujesky et al $l^{55}$ studied 366 patients diagnosed with acute PE. Increased d-dimer levels were associated with poorer 3-month all-cause mortality; however, this association was not independent of PESI. Vanni et al identified elevated plasma lactate levels $(\geq 2 \mathrm{mmol} / \mathrm{L})$ as being an independent predictor of 7-day PE-related mortality or haemodynamic collapse. ${ }^{56}$ These findings are all in need of external validation and as yet have not been shown to offer any additional benefit for stratification of very low-risk patients.

\section{Multimodality assessment}

Jimenez et $a l^{57}$ studied derivation and validation cohorts of 848 and 529 haemodynamically stable patients in a multicentre prospective study. The four independent prognostic predictors for a complicated course identified were standard cTnI, BNP, presence of DVT on USS and sPESI $>0$, with echocardiographic assessment of the RV not providing additional prognostically beneficial information. In patients with sPESI of 0 , BNP above and below $100 \mathrm{pg} / \mathrm{mL}$ was associated with a risk of 30 -day complicated course of $3.1 \%$ and $0.9 \%$, respectively, suggesting addition of BNP to sPESI could further refine risk stratification; however, mortality was zero in both groups.

Vuilleumier $e$ t $a l^{58}$ subsequently studied 230 elderly patients (>65 years) with non-severe acute PE. In this study, both NT-proBNP (threshold $300 \mathrm{pg} / \mathrm{mL}$ ) and hsTropT (threshold $14 \mathrm{pg} / \mathrm{mL}$ ) had NPVs for a 30-day combined end point of PE-related death, recurrent VTE or major haemorrhage of $>95 \%$ and outperformed PESI which was not predictive of the primary 
end point. The addition of hsTropT to PESI or Geneva Prognosis Score improved the AUC for the primary end point at ROC analysis; however, no combination of biomarker and clinical score could demonstrate a higher NPV than NT-proBNP alone (98.6\%). The authors conclude that NT-proBNP or hsTropT could be adequate alternatives to clinical risk scores in elderly patients and that the addition of hsTropT may improve the efficacy of clinical risk scores. Limitations of this study include a high exclusion rate due to missing data and the incorporation of major haemorrhage in the primary end point, which makes comparison with other studies difficult.

\section{Cardiac biomarkers in patients with radiological evidence of RV dysfunction but low-risk sPESI/PESI}

Patients who are suitable for OP management, in terms of both sPESI of 0 or PESI I/II and lack of exclusion criteria but who have had some degree of RV dysfunction demonstrated radiologically (most often on CTPA), present a common clinical dilemma. Data from four studies previously discussed above are of use in guiding an approach to this clinical problem. ${ }^{46} 485256$ In their multimodality prognostic study, Jimenez et $a l^{57}$ defined 214 patients as very low risk in terms of an sPESI of 0 and a low BNP. No 30-day complications were observed in the 12 patients (6\%) in this subgroup who had evidence of RV dysfunction on echocardiography. In their study of hsTnT and sPESI in 526 normotensive patients, Lankeit et $a l^{47}$ demonstrated RV dysfunction in $35 \%$ of the 456 patients who had undergone echocardiography. One hundred and twenty-seven patients had both sPESI of 0 and hsTnT $<14 \mathrm{pg} / \mathrm{mL}$, and none of these patients had an adverse 30-day outcome. Also, 113 of this subgroup had undergone echocardiography, with RV dysfunction being present in $17(15 \%)$. In the study of hsTnI by Hakemi et al, ${ }^{49}$ 137 of 298 patients were hsTnI-negative $(<0.012 \mathrm{ng} / \mathrm{mL})$. None of the hsTnI-negative subgroup died or required cardiopulmonary resuscitation or thrombolysis; $6 \%-8 \%$ of this subgroup had evidence of RV dysfunction on CTPA or echocardiography. In the study by Jimenez et al of RV dilatation on CTPA, an enlarged RV was observed in 533/844 patients (63\%) of whom $252(47 \%)$ had a BNP $<100 \mathrm{pg} / \mathrm{mL} .^{53}$ Although exact details regarding outcomes in patients with RV dilatation and normal BNP were not provided, as a 30-day complicated course was observed in only $\approx 4 \%(n=19)$ of all patients with a normal BNP then one can conclude that only a very small proportion of patients with RV dilatation and normal BNP levels had a complicated course.

\section{Summary}

Various validated risk prediction models suggest that a substantial proportion of patients could be managed as an OP for PE provided they are assessed as low risk and have a favourable clinical and social profile. Most of these risk models have been developed to predict 30-day mortality rather than select patients for OP management. The proportion identified as suitable depends on the selection process employed. The Geneva criteria seem to identify the largest proportion of patients as low risk compared with the other validated risk prediction tools; however, there is a consequent increase in adverse events. The Hestia criteria were designed specifically to guide selection for OP management and are the best validated of the clinical exclusion criteria in the published English-language literature but all of the clinical prediction tools seem to be able to identify a patient population at low risk of adverse events. PESI is the most extensively validated clinical prediction tool, although studies suggest that sPESI is almost equivalent and it is easier to derive. PESI and
sPESI have the advantage over Hestia of not excluding pregnant patients and not including the potentially ambiguous criterion of medical or social reason for treatment in hospital. On the other hand, PESI and sPESI exclude those with active cancer. These differences highlight grey areas and the need for experienced review. Furthermore, these risk scoring strategies have been validated against 30 -day outcome measures. These provide reassurance regarding the longer-term risk of an OP management strategy, but this time point clearly extends beyond the expected duration of an IP admission. Potentially, scores looking at shorter-term outcomes may be more relevant to early management strategies, including decisions regarding OP management.

While abnormal RV function may be of relevance in the assessment of higher-risk patients, ${ }^{5}$ the absence of radiological $\mathrm{RV}$ dysfunction cannot be used to predict an even lower risk of deterioration. There is evidence to suggest that the addition of cardiac biomarkers (BNP, NT-proBNP, hsTnI or hsTnT) may identify patients at even lower risk of a complicated course $(0 \%-1 \%)$ but this reduction in likelihood of clinical deterioration is likely to be at the expense of a reduction in the proportion of patients suitable for discharge to approximately $20 \%-30 \%$ and may also require extra resources. Laboratory cardiac biomarkers may have a role in predicting a low risk of a complicated course in patients who, despite a low-risk clinical score and the absence of exclusion criteria, have radiological or echocardiographic evidence of RV dysfunction. It is not an infrequent occurrence that troponin is requested as part of a general bundle on admission. In this context, a positive result should be treated in accordance with local trust policy and, in particular, causes for elevation other than pulmonary embolism should be considered.

An algorithm for the OP management of PE is provided in figure $1 \mathrm{~A}$ (when applying Hestia criteria) and figure $1 \mathrm{~B}$ (when applying PESI or sPESI).

\section{Evidence statement}

Clinical risk scores can identify patients at low risk of adverse outcome from PE. Evidence level 2++

In confirmed PE, PESI and sPESI are the most validated clinical 30-day risk scores. Evidence level $2++$

PESI is the most validated but sPESI may be easier to use and is just as powerful in identifying low-risk patients.

Clinical exclusion criteria (Hestia) can identify patients who are not suitable for OP management of acute PE. Evidence level $2++$

In confirmed PE, PESI (in combination with clinical/social exclusion criteria) identifies patients who have non-inferior outcomes when managed as OPs. Evidence level 1+

There is insufficient evidence to propose specific criteria to assess the risk of early bleeding on anticoagulation to identify patients needing IP admission. However, assessment criteria which identify patients with PE with low risk of early mortality appear also to select patients at lower risk of early (within 14 days) major bleeding. Evidence level 2++

Assessment of RV wall stress (BNP or NT-proBNP) or ischaemia (hsT) in addition to clinical risk scores identifies a very low-risk population. Evidence level $2++$

In identifying low-risk patients suitable for OP management, assessment of RV function with echocardiography or CT imaging does not increase the predictive power of clinical risk scores and exclusion criteria. Evidence level $2++$

In patients with RV dysfunction on echocardiography and/or CT, normal cardiac blood biomarkers identify low-risk patients. Evidence level 2++ 


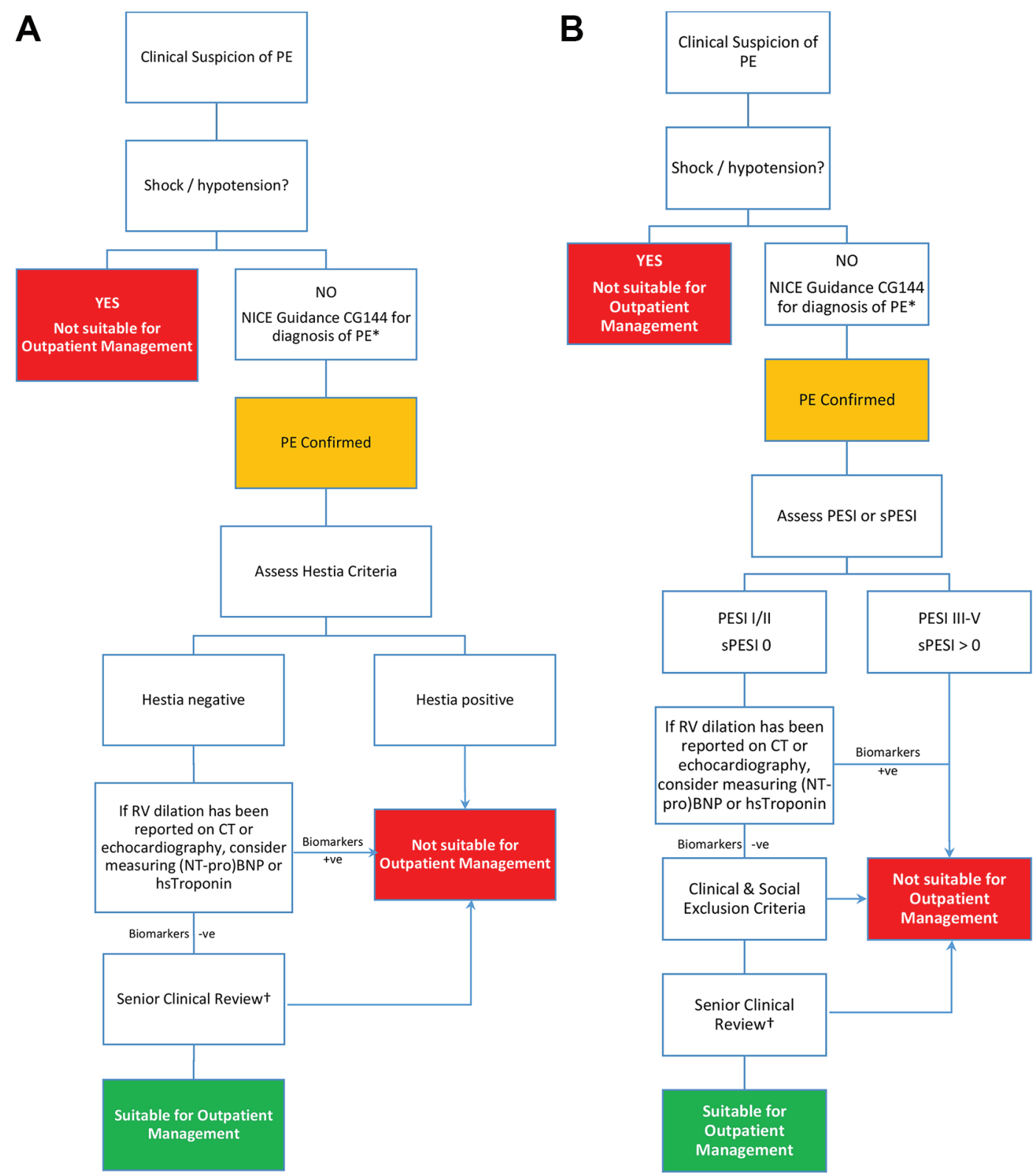

Figure 1 (A, B) Algorithm for the outpatient management of PE (A, Hestia; B, Pulmonary Embolism Severity Index (PESI) or simplified PESI (sPESI)). *If imaging cannot be undertaken same day, then patients may be considered for empirical treatment with either low molecular weight heparin or apixaban or rivaroxaban and asked to return within 24 hours for definitive diagnosis, providing they fulfil the remainder of the criteria for outpatient management. PPatients with cancer or those who are pregnant or within 6 weeks post partum may be considered for outpatient management.NICE, National Institute for Health and Care Excellence; NT-proBNP, N-terminal B-type natriuretic peptide.

Bilateral lower limb compression ultrasound, ECG, quantitative d-dimer or serum lactate measurement are not useful in identifying patients at low risk of adverse outcomes and suitable for OP management. Evidence level 2+

\section{Recommendations}

- Patients with confirmed PE should be risk-stratified using a validated clinical risk score. Grade B

- Patients in PESI class I/II, sPESI 0 or meeting the Hestia criteria should be considered for OP management of PE. Grade B

- Where PESI or sPESI is used and indicates a low risk, a set of exclusion criteria should be applied to patients being considered for OP management of confirmed PE. Grade B Exclusion criteria include:
Haemodynamic instability (HR >110; SBP $<100 \mathrm{~mm}$ $\mathrm{Hg}$; requirement for inotropes and critical care; requirement for thrombolysis or embolectomy).

- Oxygen saturations $<90 \%$ on air.

- Active bleeding or risk of major bleeding (eg, recent GI bleed or surgery, previous intracranial bleeding, uncontrolled hypertension).

- On full-dose anticoagulation at the time of the PE.

- Severe pain (eg, requiring opiates).

- Other medical comorbidities requiring hospital admission.

- Chronic kidney disease (CKD) stage 4 or 5 (eGFR $<30 \mathrm{~mL} / \mathrm{min}$ ) or severe liver disease.

- HIT within the last year and where there is no alternative to repeating heparin treatment. 
- Social reasons which may include inability to return home, inadequate care at home, lack of telephone communication, concerns over compliance, etc.

- No specific assessment of bleeding risk is required in patients who are deemed low risk by PESI/sPESI/Hestia criteria. Grade B

- Measurement of RV:LV ratio on CT or assessment of RV function on echocardiography is not obligatory for the identification of low-risk patients for OP management. Grade C

- Where RV dilatation has been identified on CT scanning or echocardiography in patients who are suitable for OP management, consider measuring laboratory cardiac biomarkers (BNP, NT-proBNP, hsTnI or hsTnT). Normal values may be used to identify low-risk patients; elevated biomarkers in this context should prompt IP admission for observation. Grade C

\section{Good practice points}

$\checkmark$ In the context of low-risk PE and an incidental finding of elevated troponin, senior review is required and alternative causes for troponin release should be considered.

\section{Research recommendation}

Prospective randomised comparison of (s)PESI against Hestia rules out criteria to determine proportion of patients who can be managed as OPs, including safety outcome. One study is currently running which will address this issue (NCT02811237).

\section{MANAGEMENT OF PATIENTS WITH SUSPECTED PE, WHERE A DIAGNOSIS HAS YET TO BE CONFIRMED}

The majority of evidence in the literature regarding the management of PE relates to confirmed PE, rather than suspected PE. However, as ambulatory care services have evolved, they have had to consider the clinical scenario in which an individual with a suspected, but not radiologically confirmed, PE could potentially be investigated/managed on an OP basis. There is a sparser evidence base on which to make recommendations in this area.

\section{Evidence review}

The evidence for managing patients with suspected PE is based on two observational cohort studies which look at differently selected populations. ${ }^{1259}$ These studies used inclusion and exclusion criteria designed to remove patients with potential haemodynamic, radiological or biochemical markers of adverse outcomes for PE, or had medical, social or practical barriers to OP investigation. One study, based in the emergency department, aimed to validate an algorithm for the diagnosis of $\mathrm{PE}$ in a selected group of patients presenting with pleuritic chest pain. ${ }^{59}$ Among 408 patients classified as intermediate or high risk of PE (as judged by a combination of Wells score and d-dimer testing) who underwent imaging, there was a low rate of diagnosed PE (5.4\%). 86.5\% were investigated as OPs with 274 patients (67.2\%) having PE diagnosed or excluded within one working day. An additional 79 patients (19.4\%) underwent OP imaging. All patients received a dose of LMWH pending imaging. Follow-up was for 3 months with significant complications defined as death or a recurrent venous thromboembolic event. Across all those recruited to the study, three patients had a recurrent venous thromboembolic event within the 3-month follow-up period and there were four deaths, one of which was attributed to PE. All those investigated as an OP had an 'uncomplicated recovery'.
A retrospective review of outcomes from an established OP PE service assessed 905 patients referred with suspected PE of whom 562 were considered at intermediate or high risk for PE and underwent imaging following a dose of LMWH. ${ }^{12}$ Same-day imaging was performed in $62.8 \%$, with $89 \%$ undergoing imaging the same or next day. The maximum time to imaging was 5 days. Eleven per cent $(96 / 905)$ had a confirmed PE with 70 managed as OPs. There were no deaths or complications related to PE in the follow-up period for those managed as OPs.

Finally, a single-centre audit of an OP care pathway for patients with suspected PE discharged from the emergency department pending imaging has been reported. ${ }^{60}$ A series of clinical exclusion criteria were applied and patients were treated with LMWH before returning for imaging within 24 hours. A total of 45 patients were managed in the OP setting, with $25 \%$ subsequently confirmed to have a PE. There were no adverse events or readmissions in any patients on this pathway.

There have been no published studies which specifically look at the role of direct oral anticoagulants in patients with suspected $\mathrm{PE}$ but this topic is covered in further detail in the next section.

\section{Evidence statement}

There are a limited number of studies in which patients with suspected PE who were identified as at low risk of adverse outcome were treated with anticoagulation prior to imaging, either same day or as an OP. Outcomes did not appear to be worse in those patients with suspected PE investigated as OP. Evidence level 2-

\section{Recommendation}

- Patients with suspected PE should, where reasonably practical, undergo investigation on the same day of presentation to exclude a diagnosis of PE. An alternative strategy of anticoagulation followed by OP imaging within 24 hours may be considered in patients with suspected PE, who have been deemed low risk and eligible for OP care as per confirmed PE. Robust systems should be in place to facilitate next day investigation and review. Grade D

\section{TREATMENT OF SUSPECTED/CONFIRMED PE IN THE OUTPATIENT SETTING}

For 40 years, the standard therapy for most patients with PE has been the administration of heparin, overlapped and followed by a VKA. ${ }^{3}$ Recently developed oral anticoagulants that are directed against factor Xa or IIa (thrombin), collectively termed DOACs, overcome some limitations of standard therapy, including the need for heparin injections in some cases and for regular dose adjustments on the basis of laboratory monitoring. ${ }^{61}$ Because of their rapid onset of action, the DOACs have the potential to enable single oral drug regimens, which can replace parenteral anticoagulants and warfarin. Both apixaban and rivaroxaban have licences to be used as a single-drug approach, whereas dabigatran and edoxaban require a lead-in period of treatment with heparin.

\section{Evidence review}

There are no studies comparing the use of DOACs against LMWH/VKA or fondaparinux in the OP setting.

There is now strong evidence that DOACs are non-inferior in the treatment of PE and have a favourable safety profile. ${ }^{62-65} \mathrm{~A}$ Cochrane review assessed the evidence for the effectiveness of 
Table 7 Key randomised trials of direct oral anticoagulants in the treatment of acute PE

\begin{tabular}{|c|c|c|c|}
\hline \multirow[b]{2}{*}{ Study (no of patients) } & \multirow[b]{2}{*}{ Treatment arm (vs heparin/warfarin) } & Efficacy & Safety \\
\hline & & \multicolumn{2}{|c|}{ (study drug vs warfarin) } \\
\hline $\begin{array}{l}\text { RE-COVER (2009) } \\
\mathrm{n}=2564\end{array}$ & LMWH $\geq 5$ days followed by dabigatran $150 \mathrm{mg}$ twice a day & Recurrent VTE or fatal PE: $2.4 \%$ vs $2.1 \%$ & Major bleeding: $1.6 \%$ vs $1.9 \%$ \\
\hline $\begin{array}{l}\text { RE-COVER II (2014) } \\
\mathrm{n}=2589\end{array}$ & LMWH $\geq 5$ days followed by dabigatran $150 \mathrm{mg}$ twice a day & Recurrent VTE or fatal PE: $2.3 \%$ vs $2.2 \%$ & $\begin{array}{l}\text { Major bleeding: } 15 \text { patients vs } 22 \\
\text { patients }\end{array}$ \\
\hline $\begin{array}{l}\text { EINSTEIN PE (2012)* } \\
\mathrm{n}=4833\end{array}$ & $\begin{array}{l}\text { Rivaroxaban } 15 \mathrm{mg} \text { twice a day for } 3 \text { weeks followed by } 20 \mathrm{mg} \\
\text { once a day }\end{array}$ & Recurrent VTE or fatal PE: $2.1 \%$ vs $1.8 \%$ & $\begin{array}{l}\text { Major or CRNM bleeding: } 10.3 \% \text { vs } \\
11.4 \%\end{array}$ \\
\hline $\begin{array}{l}\text { AMPLIFY study (2013) } \\
\mathrm{n}=5400\end{array}$ & $\begin{array}{l}\text { Apixaban } 10 \mathrm{mg} \text { twice a day for } 7 \text { days followed by } 5 \mathrm{mg} \text { twice a } \\
\text { day }\end{array}$ & Recurrent VTE or fatal PE: $2.3 \%$ vs $2.7 \%$ & Major bleeding: $0.6 \%$ vs $1.8 \%$ \\
\hline $\begin{array}{l}\text { HOKUSAI-VTE (2013) } \\
\mathrm{n}=8292\end{array}$ & $\begin{array}{l}\text { LMWH } \geq 5 \text { days followed by edoxaban } 60 \mathrm{mg} \text { once a day }(30 \mathrm{mg} \\
\text { once a day if creatinine clearance } 30-50 \mathrm{~mL} / \mathrm{min} \text { or bodyweight } \\
<60 \mathrm{~kg} \text { ) }\end{array}$ & Recurrent VTE or fatal PE: $3.2 \%$ vs $3.5 \%$ & $\begin{array}{l}\text { Major or CRNM bleeding: } 8.5 \% \\
\text { vs } 10.3 \%\end{array}$ \\
\hline
\end{tabular}

*Only EINSTEIN PE included exclusively patients with PE.

CRNM, clinically relevant non-major bleeding; LMWH, low molecular weight heparin; VTE, venous thromboembolism.

DOACS in the treatment of PE. ${ }^{66}$ The five RCTs they examined are summarised in table 7.

The Cochrane review ${ }^{66}$ concluded that there was no difference in the effectiveness of oral direct thrombin inhibitors (DTIs) and standard anticoagulation in preventing recurrent PE (OR 1.02, 95\% CI 0.50 to 2.04), recurrent VTE (OR 0.93, 95\% CI 0.52 to 1.66 ) and DVT (OR $0.79,95 \%$ CI 0.29 to 2.13 ) or causing major bleeding (OR $0.50,95 \%$ CI 0.15 to 1.68 ). The oral factor $\mathrm{Xa}$ inhibitors were no more or less effective in the prevention of recurrent VTE (OR 0.85, 95\% CI 0.63 to 1.15 ), DVT (OR 0.72, $95 \% \mathrm{CI} 0.39$ to 1.32 ) and all-cause mortality (OR $1.16,95 \% \mathrm{CI}$ 0.79 to 1.70 ) or causing major bleeding (OR $0.97,95 \%$ CI 0.59 to 1.62$)$.

Several large meta-analyses have been performed to assess the safety of DOACs in different conditions. ${ }^{616768}$ Sardar et al analysed 50 trials involving 155537 patients treated with DOACs for a variety of indications. ${ }^{68}$ Pooled analysis of all DOACs for all indications did not show a significant difference between DOACs and comparators for risk of major bleeding (OR 0.93, $95 \%$ CI 0.79 to 1.09 ). For the treatment of acute VTE or PE, DOACs were associated with significantly less bleeding (RR $0.63,95 \%$ CI 0.44 to 0.90 ).

Van Es et al performed a meta-analysis of phase III RCTs (26872 patients) comparing DOACs with VKA for the treatment of acute symptomatic VTE and also concluded that DOACs were non-inferior in terms of mortality or prevention of recurrent VTE and had a lower incidence of major bleeding (RR 0.61, $95 \%$ CI 0.45 to 0.83$)^{69}$

Gómez-Outes et al analysed 10 studies in 35019 randomised patients with VTE, of whom 14364 were treated for PE. ${ }^{61}$ They reported a lower risk of clinically relevant bleeding in those treated with a DOAC (RR 0.72 ; $95 \%$ CI 0.57 to 0.91 ) and noted there was evidence of statistical heterogeneity across the studies (table 8).

\section{OP TREATMENT OF PE USING DOACS}

The advantage of DOACs in an OP setting is that they are administered orally rather than parenterally. It is worth noting that the designs of the DOAC studies differ and only rivaroxaban and apixaban have been studied as single-drug regimens for the treatment of PE without the need for an LMWH lead-in period, although patients could receive a dose of heparin prior to diagnosis. ${ }^{63} 64$ In the RE-COVER (dabigatran) ${ }^{62}$ and Hokusai-VTE (edoxaban) ${ }^{65}$ studies, LMWH was also administered for at least 5 days. In the EINSTEIN DVT and PE studies, a cohort of 649 patients received rivaroxaban without prior treatment with heparin. ${ }^{70}$ A retrospective post-hoc analysis, comparing this cohort to those who did receive heparin $(n=3501)$, concluded that the use of prestudy heparin did not influence the efficacy and safety of rivaroxaban in patients treated for symptomatic VTE as there were no differences in terms of recurrent VTE or major bleeding.

Only one group has reported on the use of DOACs in the treatment of PE in an OP setting. Beam et al undertook a prospective observational study on 106 patients who were identified as low risk using modified Hestia criteria. ${ }^{71}$ Patients $(71$ DVT, $35 \mathrm{PE}$ ) were discharged from the emergency department with a prescription for $15 \mathrm{mg}$ rivaroxaban twice per day for 21 days followed by $20 \mathrm{mg}$ daily as per its licence. Patients could receive a single dose of $1 \mathrm{mg} / \mathrm{kg}$ enoxaparin prior to discharge or one $15 \mathrm{mg}$ dose of rivaroxaban by mouth, depending on the immediate availability of rivaroxaban. There were no major bleeding events or episodes of recurrent VTE during the period of anticoagulation. The same group published further data on a larger cohort collected of an extended period of time according to the same protocol, including an additional 32 patients with PE from the original study. There were no additional PE recurrences nor major bleeding events in the PE group. ${ }^{72}$

\section{DOACs and impact on LOS}

Single-dose DOAC regimens offer the advantage of a faster time to reach therapeutic anticoagulation, which is attained after the first dose, in contrast to traditional VKAs which require at least

Table 8 Relative risk of major and non-major bleeding using direct oral anticoagulants compared with vitamin $\mathrm{K}$ antagonists

\begin{tabular}{lll}
\hline Study & Drug & $\begin{array}{l}\text { Risk ratio of major and } \\
\text { clinically relevant non- } \\
\text { major bleeding events }\end{array}$ \\
\hline RE-COVER (2009) & Dabigatran & $0.64(0.48-0.85)$ \\
\hline RE-COVER II (2014) & Dabigatran & $0.63(0.47-0.86)$ \\
\hline EINSTEIN DVT (2010) & Rivaroxaban & $1.00(0.80-1.25)$ \\
\hline EINSTEIN PE (2012) & Rivaroxaban & $0.91(0.77-1.07)$ \\
\hline AMPLIFY Study (2013) & Apixaban & $0.44(0.36-0.55)$ \\
\hline HOKUSAI-VTE (2013) & Edoxaban & $0.83(0.72-0.95)$ \\
\hline
\end{tabular}

Risk ratio of major and clinically relevant non- 
5 days of bridging with a parenteral anticoagulant and an INR $>2$ before the desired anticoagulation is achieved. Therefore, these regimens have the potential to reduce LOS and/or facilitate early discharge.

Van Bellen et al reviewed the length of initial hospitalisation in patients presenting with either symptomatic DVT or PE using hospitalisation records from the EINSTEIN-DVT and PE trials. ${ }^{73}$ Overall, 90\% (4328/4821) of EINSTEIN PE patients were admitted to hospital. The proportion of hospitalised patients with a LOS of five or fewer days receiving rivaroxaban was $45 \%$ compared with 33\% for enoxaparin/VKA in patients with PE. Stays of 6-10 days were observed in 39\% of rivaroxaban-treated patients compared with $46 \%$ of enoxaparin/VKA-treated patients for PE. LOS was significantly shorter in the rivaroxaban group compared with the enoxaparin/VKA group across all analyses performed $(p<0.0001)$. As this was an international study, there were very significant variations in the observed admission rates and LOS.

A post-hoc analysis of hospitalisation and LOS in the North American subset of patients from the EINSTEIN study evaluated LOS using investigator records of dates of admission and discharge. ${ }^{74}$ Of 382 patients hospitalised, 321 (84\%) had acute symptomatic PE; few patients with DVT required hospitalisation. Similar rates of patients with VTE were hospitalised in the rivaroxaban and enoxaparin/VKA treatment groups, $189 / 405(47 \%)$ and 193/401 (48\%), respectively. In hospitalised patients with VTE, rivaroxaban treatment produced a 1.6-day mean reduction in LOS (median $=1$ day) compared with enoxaparin/VKA (mean $=4.5$ vs 6.1 ; median $=3$ vs 4 ). This was primarily driven by the 1.7 -day mean reduction in hospital LOS for patients with PE treated with rivaroxaban $(n=162)$ versus enoxaparin/VKA $(n=159$; mean $=4.5$ vs 6.2 ; median $=3$ vs $4 ; \mathrm{p}=0.0002$ ).

Another post-hoc analysis was undertaken in the Japanese cohort of the EINSTEIN DVT and PE studies which examined the length of hospital stay in patients with PE and/or DVT receiving rivaroxaban compared with Japanese standard therapy. ${ }^{75}$ In Japan, the standard of care for the treatment of PE and/or DVT consists of intravenous unfractionated heparin followed by warfarin. Analyses were conducted in the intentionto-treat population of 97 participants. The median LOS in rivaroxaban patients was 10 days compared with 15 days for patients on standard therapy $(p=0.016)$. The limitations of the study include a non-standard dose of rivaroxaban $15 \mathrm{mg}$ twice daily for 21 days followed by $15 \mathrm{mg}$ once daily, the small sample size and the generalisability of the findings to a UK setting.

A multicentre, retrospective, observational cohort study of 158 patients, admitted with a primary discharge diagnosis of PE, compared 82 patients treated with enoxaparin and warfarin and 76 treated with rivaroxaban. ${ }^{76}$ The median LOS was 2.7 days shorter for those treated with rivaroxaban (1.8 days (IQR 1.2 to 3.7) vs 4.5 days (IQR 2.7 to 5.9) enoxaparin plus warfarin $(p<0.001))$. The time from initial dose of anticoagulant to time of discharge was also significant; the median interval in the warfarin plus enoxaparin group was 3.9 days while the median interval was 0.9 days in the rivaroxaban group $(\mathrm{p}<0.001)$. This study has limitations as there may be confounding factors for clinicians choosing one regimen over another.

Masotti et al retrospectively analysed data on patients discharged with PE from five hospitals in the Florence district, Italy, over a 4 -year period. ${ }^{77}$ They found that mean and median LOS in patients on DOACs was significantly lower than in patients on other anticoagulant treatments.

\section{DOACs for the treatment of suspected PE}

There are no studies that have been undertaken specifically to examine the safety or efficacy of managing suspected PE with a DOAC. The only studies to report on the safety and efficacy of OP management of suspected PE, when imaging could not be performed immediately, both used LMWH while a diagnosis was pending. 1259

\section{Evidence statement}

DOACs are non-inferior compared with LMWH followed by VKAs for the treatment of PE in terms of recurrent VTE and all-cause mortality. Evidence level $1++$

DOACs are associated with a lower risk of clinically relevant bleeding in patients treated for VTE. Evidence level $1++$

Rivaroxaban and apixaban can be used as part of a singledrug regimen without the need for an LMWH lead-in period. Evidence level $1++$

Treatment of PE with DOACs may be associated with a shorter LOS in hospital. Evidence level 2-

\section{Recommendations}

- Patients with confirmed PE being treated in the OP setting should be offered treatment with either LMWH and dabigatran, LMWH and edoxaban or a single-drug regimen (apixaban or rivaroxaban). Grade A

- Patients with suspected PE being treated in the OP setting may be treated with apixaban or rivaroxaban pending diagnosis as an alternative to LMWH. Grade D

\section{Good practice point}

$\checkmark$ Using a single DOAC in a pathway is preferred to minimise potential confusion over dosing and administration.

\section{ASSESSING PATIENTS TRANSITIONING FROM IP CARE TO EARLY DISCHARGE/OP CARE}

With the advent of DOACs, patients may be considered early for discharge rather than once therapeutic anticoagulation has been achieved with VKAs. Tools and strategies can assist clinical judgement in when it may be appropriate to discharge patients.

\section{Evidence review}

Only one study was identified as suitable for inclusion in this section which was conducted in Spain. Moores et $a l^{78}$ performed a retrospective cohort study of 304 patients who were classified at the time of diagnosis into PESI class III (intermediate risk). Seventy-seven per cent were aged $>65$ years. They recalculated the PESI again at 48 hours following diagnosis (PESI-48) and reclassified the patients accordingly. They also calculated the sPESI at 48 hours (sPESI-48) and stratified patients as low risk (score 0) or high risk (score $\geq 1$ ). The authors compared the outcomes (all-cause mortality days 2-30, recurrent VTE and major bleeding) in those patients who were reclassified as low risk (PESI-48 class I or II or sPESI-48 score 0 ) with those who remained high risk.

The PESI-48 score reclassified 27.3\% (83/304) (95\% CI $22.3 \%$ to $32.3 \%$ ) as low risk. This was predominantly due to changes in physiological parameters such as heart rate, SBP, temperature, respiratory rate or oxygen saturation. Mortality at 30 days in the low-risk PESI-48 group was $1.2 \%(95 \% \mathrm{CI}$ $0 \%$ to $3.5 \%$ ) versus $8.3 \%$ (95\% CI $4.5 \%$ to $12.1 \%$ ) in those who remained PESI class III. Five per cent of patients were reclassified as high risk (class IV or V) when the PESI-48 score was recalculated and in this subgroup 30-day mortality was $50 \%$ (95\% CI $25.5 \%$ to $74.5 \%)$. 
sPESI was retrospectively calculated at admission and 250 $(82 \%)$ patients were deemed to be high risk at this point. Recalculation of sPESI-48 in these 250 was subsequently reclassified $15.2 \%$ as low risk. Mortality at 30 days was $0 \%$ in the low-risk sPESI-48 group compared with $12.3 \%$ (95\% CI $7.8 \%$ to $16.7 \%$ ) in those who remained high risk.

Adverse events were similar in both PESI-48 and sPESI-48 groups $(2.4 \%$ (95\% CI $0 \%$ to $5.7 \%)$ and $4.8 \%$ (95\% CI $0.2 \%$ to 9.3\%), respectively).

\section{Evidence statement}

In patients who are admitted in PESI class III, a PESI class of I or II or sPESI score of 0 at 48 hours identifies a low-risk population who can be considered for early discharge. Those who are not reclassified as low risk have a higher risk of adverse outcome. Evidence level 2+

\section{Recommendation}

- Patients who have been admitted with an intermediate risk PE (PESI class III) can be considered for early discharge when they meet the criteria for low risk (PESI class I/II or sPESI score 0). Grade C

\section{Good practice points}

$\checkmark$ Those with PESI-48 class III or sPESI-48 score of $>0$ are considered to be at higher risk of adverse outcome and senior review is necessary prior to discharge; PESI and sPESI may remain elevated due to non-reversible factors (eg, cancer, age) which should be taken into consideration when using clinical judgement.

$\checkmark$ Consideration should be given to repeating assessment of RV function with echocardiography or biomarkers in those admitted with RV dysfunction or biomarker elevation at baseline.

\section{LEVEL OF SENIORITY OF REVIEW Evidence review}

There was no evidence in the literature to help determine the optimal seniority of staff assessment required within OP care pathways. The guideline group reviewed the statement produced by the Royal College of Emergency Medicine on consultant sign-off for non-traumatic chest pain and conditions of similar risk. This stated that where patients could not be reviewed by an emergency medicine consultant, that they should be reviewed by a senior trainee in emergency medicine (ST4 or above) or by a staff grade or similar substantive career grade doctor with sufficient emergency department experience to be designated to undertake this role by the emergency medicine consultant medical staff. Due to greater experience in the medical specialties from core medical training prior to entry into the specialist trainee grade in acute and specialist medicine, compared with emergency medicine, the group felt that ST3 and above would provide an adequate level of seniority within these specialties, which could be applied to OP management of PE. ${ }^{79}$ The group also felt that advanced nurse practitioners or clinical nurse specialists could be trained and designated to undertake this role with consultant support. Finally, the group concluded that the clinical review should also formally take in to consideration alternative diagnoses in the case of suspected PE.

\section{Good practice points}

$\checkmark \quad$ Patients with confirmed or suspected PE should be reviewed by a consultant prior to discharge on an OP PE pathway. If no consultant is available, then patients may be reviewed by a senior trainee (ST3 or above; ST4 in the case of emergency medicine) by a staff grade or similar substantive career grade doctor, advanced nurse practitioner or clinical nurse specialist designated to undertake this role within the department with consultant advice available.

$\checkmark \quad$ If patients are on an OP pathway for suspected PE and being considered for discharge and scanning the following day, a local protocol should be in place to guide a full cardiorespiratory assessment to exclude other causes for symptoms (including full history, examination, ECG and chest radiograph), including risk assessment.

\section{FOLLOW-UP OF PATIENTS SPECIFIC TO THOSE MANAGED IN THE OP SETTING}

In all of the studies detailed below, follow-up arrangements were a fixed part of the study protocols and as such were not designed to address the question of how best to follow-up patients; thus, recommendations are extrapolated from assessment of procedural safety within OP studies. The majority of these studies are performed as service evaluations, although one OP protocol within a RCT has also been evaluated.

Only aspects specific to an OP management strategy are discussed.

\section{Evidence review}

\section{Intensity of initial follow-up}

Several studies involving patients discharged home within 24 hours of the diagnosis of acute PE have been published. In the RCT of Aujesky et al, participants were followed up after discharge by a protocol of daily telephone calls for the first week. ${ }^{8}$ This was followed by calls on days $14,30,60$ and 90 by a specialist nurse. General enquiries were made regarding symptoms of recurrence and bleeding complications, with advice to attend the emergency department for review as needed. Outcomes were reported at 90 days in the OP group as recurrent VTE (0.6\%), major bleeding (0.6\%) and all-cause mortality $(0.6 \%)$. No significant difference was found for any outcome when compared against IP rates, implying the procedural safety of this follow-up protocol. (The power of this study to detect non-inferiority of OP vs IP management was $80 \%$ with a one-sided $\alpha$ of 0.05 at a $4 \%$ margin.)

Agterof et al studied 152 prospective patients with normal NT-proBNP levels. ${ }^{13}$ Patients received written instructions regarding readmission, if specific symptoms or signs should develop, and a 24-hour emergency contact number. They were also contacted by telephone on days 2 and 4 and were clinically reviewed on day 10 . No deaths, major bleeding or recurrence episodes occurred within the first 10 days. Seven (4.6\%) patients required hospitalisation within the first 10 days, although over half of these (4/7) were deemed unrelated to the diagnosis of PE. The remaining three cases were admitted for further review as suspected complications of PE but underwent no additional procedures or changes in anticoagulant therapy. All three were discharged safely after a stay of 2-5 days.

In a later study, Zondag et al prospectively enrolled and ambulated 247 patients with proven PE and no prespecified exclusion criteria. ${ }^{36}$ Patients were reviewed as an OP on day 7 and received a telephone contact at 6 weeks. No patients died during the first week of follow-up but two patients had died (deemed unrelated to PE) within the first 30 days. At 3 months, a single patient had suffered a major bleeding episode and VTE recurrence had been detected in five patients. 
In two retrospective cohort studies, similar patients were followed up by daily phone calls and had access to a 24/7 helpline. ${ }^{80}$ No adverse events were reported within these cohorts, although it should be noted that all patients had an average IP stay initially of 3-4 days.

A further recent prospective service evaluation described immediate discharge from the emergency department on diagnosis of PE, with telephone follow-up after 1-2 days followed by formal clinic review at 3 weeks. ${ }^{71}$ No adverse events (recurrence or major bleeding episodes) were described in this cohort during the treatment period. Of note, these patients were discharged and maintained on a DOAC rather than VKA.

Two additional prospective cohort studies describing experience within an OP assessment clinic for the work-up of suspected PE did not provide clear details regarding the follow-up procedure. ${ }^{12} 59$ Within all studies, OP anticoagulation was managed either by a primary care physician or dedicated anticoagulant clinic.

\section{Platelet count monitoring}

No evidence was found suggesting a clinical benefit from regular platelet count monitoring in patients discharged with a diagnosis of acute PE. In addition, the national British Committee for Standards in Haematology guidance has recently clarified the lack of benefit of routine platelet count monitoring for medical patients prescribed heparin. ${ }^{82}$ It should be noted that the same guidance suggests an initial baseline platelet count in all patients receiving heparin.

\section{Malignancy screening}

Detailed review of the evidence for malignancy screening is out of scope for this guideline since it is not specific to OP management of PE. However, no evidence was found supporting the effectiveness of routine malignancy screening specifically within low-risk patients. The NICE and the ESC have previously published guidance on screening for occult malignancy in unprovoked venous thromboembolic disease. ${ }^{283}$ Both guidelines emphasise the importance of early assessment for underlying malignancy so that further investigations can be appropriately tailored. NICE suggests consideration of extensive screening (including abdomino-pelvic CT) in addition to routine limited screening, based on the randomised controlled SOMIT study. ${ }^{84}$ The subsequent ESC guidelines have not supported routine extensive screening, evidencing their conclusion by the larger prospective TROUSSEAU study which compared 288 patients undergoing limited screening with 342 patients who also underwent extensive screening. ${ }^{85}$ A recently published RCT of 854 patients failed to demonstrate the benefit of routine abdomino-pelvic CT compared with more limited screening (which included cervical, prostate and breast cancer screening). ${ }^{86}$

\section{Evidence statement}

Contact and safety-netting in the early stages of OP management via initial telephone follow-up, written information and/or clinical assessment appears to facilitate a safe and effective strategy for OP care, resulting in low rates of complication, recurrence or major haemorrhage. Evidence level $2++$

\section{Recommendations}

- Patients with confirmed PE who are eligible for OP care should be provided with verbal and written information on the signs and symptoms of recurrence, major bleeding and additional complications. Individual centres should also provide an appropriate point of contact in the event of complications or concerns, both in and out of hours. Grade B

- Patients should have a formal review (telephone/face to face) at least once during the first week after discharge to ensure therapeutic compliance along with the absence of complications. Grade B

- Hospitals should have local protocols and pathways in place for follow-up of all patients with PE, whether treated as an IP or OP. This should include assessment of ongoing symptoms (with further directed investigation as appropriate) and consideration of optimal duration/modality of anticoagulation. Grade D

\section{Good practice points}

$\checkmark$ Consider initial assessment of provoking risk factors for the index PE at an early stage, for example, immobility, surgery, cancer, intercurrent illness, etc, since this will determine duration of anticoagulation. Screening policies for malignancy are out of scope for this guideline, but when screening investigations are performed, a mechanism should be in place to review results within a prompt time frame.

$\checkmark$ Follow-up of PE should be performed by clinicians with a special interest in VTE.

\section{Research recommendation}

Further studies evaluating the role of technology for remote monitoring, such as virtual consultations and data gathering, are needed.

\section{Management of PE in the OP setting in specific circumstances Pregnancy/puerperium}

\section{Evidence review}

Pregnancy and the puerperium increase the risk of VTE fourfold to sixfold. PE is the leading direct cause of maternal mortality in the UK. ${ }^{87}$ Rates of death from thrombosis (the majority of which are from PE) per 100000 maternities were 1.01 (95\% CI 0.65 to 1.50 ) in 2011-2013 and 0.85 (95\% CI 0.52 to 1.32) in 2012-2014. The UK incidence of antenatal PE calculated in the UK obstetric surveillance system was 1.3 per 100000 maternities, ${ }^{88}$ and the absolute incidence of VTE in pregnancy and the puerperium from a population-based cohort study was 107 per 100000 person-years. ${ }^{89}$

Every woman should be risk assessed for VTE in early pregnancy and again if admitted or during any intercurrent illness. ${ }^{90}$ Therefore, those presenting with suspected PE but not subsequently confirmed should be risk assessed to ensure they are offered prophylactic LMWH (not DOACs, see below) if they fulfil the Royal College of Obstetricians and Gynaecologists (RCOG) criteria.

Updated guidelines for the acute management of VTE in pregnancy have recently been published by the RCOG. These recommend against the use of $\mathrm{d}$-dimer testing and pre-test probability assessment in pregnancy. ${ }^{91}$ They also highlight that DOACs are likely to cross the placenta and should be avoided in the antenatal period.

No specific evidence was found regarding the OP management of pregnant or postpartum women with suspected or confirmed PE or DVT. Given that pregnancy is associated with a physiological fall in blood pressure, a rise in heart rate, a subjective feeling of breathlessness in many (as well as a reduction in $\mathrm{PCO}_{2}$ and increase in $\mathrm{PO}_{2}$ ), PESI and sPESI are likely to overestimate the risk to the pregnant woman. In studies using the Hestia criteria, 
pregnancy was itself an exclusion to OP management. In the literature, there is no consensus regarding OP management of PE in pregnancy or the puerperium. Pregnant and postpartum women should not be excluded from OP care pathways. Clear communication between the medical, haematological and obstetric teams with documentation regarding follow-up and responsibility for ongoing investigation and care is imperative.

\section{Good practice points}

$\checkmark$ All pregnant and postpartum women presenting with suspected PE or confirmed PE should be reviewed by a consultant and discussed with maternity services prior to discharge.

$\checkmark$ Outpatient care pathways may be considered for suspected or confirmed PE in pregnancy and/or the postpartum period.

$\checkmark$ Clinical risk scores derived for non-pregnant patients, such as PESI/sPESI, should not be used in pregnant women.

$\checkmark$ DOACs or VKAs should not be used in pregnant patients with suspected or proven PE.

\section{Research recommendation}

Studies addressing the safety and efficacy of OP care pathways for pregnant and postpartum patients with suspected or confirmed PE are needed.

\section{Cancer}

VTE is an important cause of morbidity and mortality in patients with cancer and the occurrence of VTE is a poor prognostic factor. ${ }^{92}$ Patients may also present incidentally without symptoms of PE during staging scans, although these patients appear to have similar long-term outcomes to those with diagnosed with symptoms. ${ }^{93}$

The risk of developing VTE may be influenced by cancer type, stage and the administration of systemic cancer treatments. The approximate annual incidence of VTE is estimated to be 1 in 200 patients with cancer. ${ }^{94}$

It is recognised that patients with malignancy are at approximately threefold increased risk of recurrent VTE, despite adequate anticoagulation, ${ }^{95}$ but also have a sixfold increased risk of treatment-related major bleeding. ${ }^{96}$

\section{Evidence review}

In the majority of patients with cancer, the application of recognised scoring systems for mortality risk, including PESI and sPESI, will result in patients falling into high-risk mortality groups, although this may be related to non-PE-related mortality. Since PESI and sPESI were not specifically designed as tools to risk-stratify patients for OP management, this necessitates an alternative approach to selecting patients for OP management with active cancer. One study developed a score from an internal derivation and validation cohort of pateints with cancer and acute PE from the RIETE registry which was then validated in 261 patients in an external cohort; the score included six variables: age $>80$ years, heart rate $\geq 110 / \mathrm{min}, \mathrm{SBP}<100 \mathrm{~mm} \mathrm{Hg}$, bodyweight $<60 \mathrm{~kg}$, recent immobility and presence of metastases. ${ }^{97}$ Thirty-day mortality in the low-risk cohort (score $=0$, $22.2 \%$ of patients) was $4.4 \%$ compared with $29.9 \%$ in the high-risk group and the the external validation cohort, 30-day mortallity was $0 \%$ in the low-risk group. This score has not been applied prospectively in OP management of patients with cancer-associated PE. Alternative scoring systems specifically for use in patients with cancer, such as the POMPE-C score, ${ }^{98} 99$ require further validation.
Eight studies were identified that included patients with cancer and VTE treated as OPs, including five prospective cohort studies and ${ }^{13} 34$ 100-102 three retrospective cohort studies. ${ }^{35} 103104$ Three studies included only patients with cancer and concurrent VTE, two included DVT/PE ${ }^{102103}$ and one included only patients with cancer and diagnosed PE. ${ }^{100}$

Across all studies, 686 patients with underlying malignancy and PE were included. In total, 306 patients with malignancy and diagnosed PE were managed as OPs or with early discharge.

Study heterogeneity, including time to discharge, treatment regimens and follow-up period, together with the absence of results specifically relating to patients with cancer in population-level studies, are significant limitations in the available evidence. Thus, it is difficult to comment on the safety of OP management of diagnosed PE in patients with cancer.

These studies did not use risk assessment tools, such as PESI/ sPESI, to identify patients suitable for OP treatment. Patients were considered for home treatment if they had no exclusion criteria. Exclusion criteria for OP treatment in cancer patients with PE, although not identified as such in eight studies, mirrored the Hestia criteria (which does not include cancer as an exclusion). ${ }^{30}$

Treatment regimens included $\mathrm{LMWH} /$ warfarin or $\mathrm{LMWH}$ alone, reflecting the year of study publication. There is currently no published evidence to support the use of DOACs over LMWH in patients with cancer.

Study sites included North America and Western Europe (Italy, Spain and the Netherlands). No studies from the UK were identified.

\section{Evidence statement}

The use of sPESI automatically excludes patients with cancer from OP management of PE and use of PESI is likely to result in automatic exclusion, limiting their utility. Evidence level 4 The Hestia criteria do not exclude patients with cancer from OP management of PE. Evidence level 2+

Specific clinical risk scores for patients suffering with cancer require further validation. Evidence level $2++$

\section{Recommendation}

- The Hestia criteria may be used to evaluate patients with active cancer for suitability for OP management of PE. Grade D

\section{Good practice points}

$\checkmark$ Patients with active cancer should be reviewed by a consultant prior to discharge given the higher risk of 30-day mortality.

$\checkmark$ Patients with incidental PE should be managed in the same way with respect to OP management as those with symptomatic PE.

\section{Research recommendation}

Further studies are needed to validate risk stratification tools specific to patients with cancer.

\section{Intravenous drug use}

Intravenous drug use is a recognised risk factor for venous thrombosis, particularly in young adults. ${ }^{105-107}$ While DVT and recurrent DVT is common in intravenous drug users (IVDU), symptomatic PE, either in isolation or in association with DVT, appears to be relatively uncommon. ${ }^{107-109}$ 


\section{Evidence review}

No evidence specific to the management of PE in IVDU was identified. Several retrospective cohort studies, primarily reporting on IVDU patients with DVT, documented the challenges in managing these patients. ${ }^{106} 109$ IVDU patients, more so than non-IVDU patients with DVT, required admission for investigation and treatment because of their chaotic lifestyles, impaired cognition and lack of finance to return for OP visits. While these cohort studies suggest IVDU is a clear risk factor for DVT, none have commented on anticoagulant-related bleeding risk in this patient category. However, IVDU has been identified as an independent risk factor for subtherapeutic INR in patients treated with warfarin for multiple indications in a cohort of patients with $\mathrm{HIV}^{110}$

\section{Good practice point}

$\checkmark$ Intravenous drug abusers with suspected PE should be admitted for further investigation and management.

\section{PATIENT INFORMATION AND SUPPORT NEEDS}

\section{Evidence review}

There have been no published studies which specifically look at the information and support needs of patients being investigated for PE.

Evidence for the information and support needs of patients with PE is based on one retrospective observational cohort study from the USA. ${ }^{80}$ The study investigators speculated that many uninsured patients with VTE lacked sufficient disease and treatment information and lacked surveillance and timely access to medical care following hospitalisation. They hypothesised that, through (1) targeted education of patients and providers, (2) coordination of timely follow-up for at-risk patients and (3) posthospital monitoring, they could achieve standardised care for all acute DVT and low-risk patients with PE. As a result, the aims were to decrease hospital LOS and produce fewer return visits and readmissions. A total of 241 patients entered the VTE clinical care pathway: 107 with DVT (44.4\%) and 134 with a PE (55.6\%). Within the pathway, uninsured patients with VTE were admitted at a lower rate than insured patients (65.9 vs $79.1 \%$; $p=0.032)$. LOS decreased from 4.4 to 3.1 days $(p<0.001)$ for admitted patients with VTE and from 5.9 to 3.1 days among uninsured patients $(p=0.0006)$. The rate of emergency department revisits at 30 days was $11 \%$ but declined from $17.9 \%$ to $13.6 \%$ in patients without health insurance $(p=0.593)$. Fewer patients treated within the pathway were readmitted $(5.8 \%)$ compared with historical patients $(9.4 \%, \mathrm{p}=0.254)$. Healthcare costs were significantly decreased.

By implementing an interdisciplinary, clinical pathway and standardised care for patients with VTE, the authors demonstrated significant reductions in health resource utilisation and cost, particularly among uninsured patients. This supports a model for improving transitional care coordination with local community health clinics and delivering care to vulnerable populations.

No UK-based evidence for patient information and support needs is available; however, the Leicester model demonstrated potential savings of an ambulatory care for management of PE in an UK teaching hospital setting. ${ }^{12}$

\section{Evidence statement}

Standardised VTE pathways, which include patient information, may reduce healthcare resource use, particularly in uninsured patients in the USA. Evidence level 2-

\section{Recommendation}

- Written patient information and education should be integral to OP PE pathways. Grade D

\section{Good practice point}

$\checkmark$ Succinct written information should be provided to the patient on discharge, using non-technical language and including telephone numbers/email addresses for advice on dealing with any subsequent changes in the patient's condition. An example, from Sheffield Teaching Hospitals, is included in online supplementary web appendix 3. The information material produced by the thrombosis charity Thrombosis UK (http://www.thrombosisUK.org) may also prove helpful.

\section{SUMMARY OF RESEARCH RECOMMENDATIONS}

Research is required to enhance the evidence base regarding patient experience and cost effectiveness.

Prospective randomised comparison of (s)PESI against Hestia rules out criteria to determine proportion of patients who can be managed as OPs, including safety outcome. One study is currently running which will address this issue (NCT02811237)

Further studies evaluating the role of technology for remote monitoring, such as virtual consultations and data gathering, are needed.

Studies addressing the safety and efficacy of outpatient care pathways for pregnant and postpartum patients with suspected or confirmed PE are needed.

Further studies are needed to validate risk stratification tools specific to patients with cancer.

Acknowledgements The British Thoracic Society and the Guideline Group members are particularly grateful to Dr Rodney Hughes and Dr Matthew Fay who contributed to the early work of the guideline group; the BTS Standards of Care Committee.

Competing interests All members of the Guideline Group made declarations of interest in line with BTS Policy and further details can be obtained on request from BTS. Guideline Development Group members are listed in appendix 1.

(C) Article author(s) (or their employer(s) unless otherwise stated in the text of the article) 2018. All rights reserved. No commercial use is permitted unless otherwise expressly granted.

\section{REFERENCES}

1 Otero R, Uresandi F, Jiménez $\mathrm{D}$, et al. Home treatment in pulmonary embolism. Thromb Res 2010;126:e1-e5.

2 NICE. Venous thromboembolic diseases: the management of venous thromboembolic diseases and the role of thrombophilia testing, 2012. CG144 2012.

3 Kearon C, Akl EA, Comerota AJ, et al. Antithrombotic therapy for VTE disease: antithrombotic therapy and prevention of thrombosis, 9th ed: American College of chest physicians evidence-based clinical practice guidelines. Chest 2012:141:e419S-94.

4 Kearon C, Akl EA, Ornelas J, et al. Antithrombotic Therapy for VTE Disease: CHEST guideline and expert panel report. Chest 2016;149:315-52.

5 Konstantinides SV, Torbicki A, Agnelli G, et al. 2014 ESC guidelines on the diagnosis and management of acute pulmonary embolism. Eur Heart J 2014;35:3033-69.

6 Elf JE, Jögi J, Bajc M. Home treatment of patients with small to medium sized acute pulmonary embolism. J Thromb Thrombolysis 2015;39:166-72.

7 Davies CWH, Wimperis J, Green ES, et al. Early discharge of patients with pulmonary embolism: a two-phase observational study. Eur Respir J 2007:30:708-14.

8 Aujesky D, Roy P-M, Verschuren F, et al. Outpatient versus inpatient treatment for patients with acute pulmonary embolism: an international, open-label, randomised, non-inferiority trial. The Lancet 2011;378:41-8.

9 Uresandi F, Otero R, Cayuela A, et al. A Clinical prediction rule for identifying shortterm risk of adverse events in patients with pulmonary thromboembolism. Archivos de Bronconeumología 2007;43:617-22.

10 Zondag W, Kooiman J, Klok FA, et al. Outpatient versus inpatient treatment in patients with pulmonary embolism: a meta-analysis. Eur Respir J 2013;42:134-44

11 Piran S, Le Gal G, Wells PS, et al. Outpatient treatment of symptomatic pulmonary embolism: a systematic review and meta-analysis. Thromb Res 2013;132:515-9. 
12 Vali Y, Ladwa R, Bailie E, et al. Investigating and managing suspected pulmonary embolism in an outpatient setting: the Leicester experience. Thorax 2015;70:291-3.

13 Agterof MJ, Schutgens RE, Snijder RJ, et al. Out of hospital treatment of acute pulmonary embolism in patients with a low NT-proBNP level. J Thromb Haemost 2010;8:1235-41

14 Aujesky D, Obrosky DS, Stone RA, et al. Derivation and validation of a prognostic model for pulmonary embolism. Am J Respir Crit Care Med 2005;172:1041-6.

15 Aujesky D, Roy P-M, Le Manach CP, et al. Validation of a model to predict adverse outcomes in patients with pulmonary embolism. Eur Heart J 2006;27:476-81.

16 Aujesky D, Perrier A, Roy P-M, et al. Validation of a clinical prognostic model to identify low-risk patients with pulmonary embolism. J Intern Med 2007;261:597-604.

17 Donze J, Le Gal G, Fine MJ, et al. Prospective validation of the Pulmonary Embolism Severity Index A clinical prognostic model for pulmonary embolism. Thrombosis and Haemostasis 2008:100:943-8.

18 Jiménez D, et al. Simplification of the Pulmonary Embolism Severity Index for Prognostication in Patients With Acute Symptomatic Pulmonary Embolism. Arch Intern Med 2010;170:1383-9.

19 Wicki J, Perrier A, Perneger TV, et al. Predicting adverse outcome in patients with acute pulmonary embolism: a risk score. Thromb Haemost 2000;84:548-52.

20 Nendaz MR, Bandelier P, Aujesky D, et al. Validation of a risk score identifying patients with acute pulmonary embolism who are at low risk of clinical adverse outcome. Thromb Haemost 2004;91:1232-6.

21 Aujesky D, Obrosky DS, Stone RA, et al. A prediction rule to identify low-risk patients with pulmonary embolism. Arch Intern Med 2006;166:169-75.

22 Agterof MJ, Schutgens REG, Moumli N, et al. A prognostic model for short term adverse events in normotensive patients with pulmonary embolism. Am J Hematol 2011;86:646-9.

23 Agterof MJ, van Bladel ER, Schutgens REG, et al. Risk stratification of patients with pulmonary embolism based on pulse rate and D-dimer concentration. Thromb Haemost 2009;102:683-7

24 Barra S, Paiva L, Providência R, et al. LR-PED rule: low risk pulmonary embolism decision rule - a new decision score for low risk pulmonary embolism. Thromb Res 2012;130:327-33.

25 Jakobsson C, Jiménez D, Gómez V, et al. Validation of a clinical algorithm to identify low-risk patients with pulmonary embolism. J Thromb Haemost 2010;8:1242-7.

26 Maestre A, Trujillo-Santos J, Riera-Mestre A, et al. Identification of low-risk patients with acute symptomatic pulmonary embolism for outpatient therapy. Ann Am Thorac Soc 2015:12:1122-9.

27 Erkens PMG, Gandara E, Wells PS, et al. Does the pulmonary embolism severity Index accurately identify low risk patients eligible for outpatient treatment? Thromb Res 2012;129:710-4

28 Jiménez D, Yusen RD, Otero R, et al. Prognostic models for selecting patients with acute pulmonary embolism for initial outpatient therapy. Chest 2007;132:24-30.

29 Kabrhel C, Okechukwu I, Hariharan P, et al. Factors associated with clinical deterioration shortly after PE. Thorax 2014;69:835-42.

30 Zondag W, Mos IC, Creemers-Schild D, et al. Outpatient treatment in patients with acute pulmonary embolism: the Hestia Study. J Thromb Haemost 2011;9:1500-7.

31 den Exter PL, Zondag W, Klok FA, et al. Efficacy and safety of outpatient treatment based on the hestia clinical decision rule with or without N-Terminal Pro-Brain Natriuretic Peptide Testing in Patients with Acute Pulmonary Embolism. A Randomized Clinical Trial. Am J Respir Crit Care Med 2016;194:998-1006.

32 Zondag W, Hiddinga BI, Crobach MJT, et al. Hestia criteria can discriminate highfrom low-risk patients with pulmonary embolism. Eur Respir J 2013;41:588-92.

33 Rodríguez-Cerrillo M, Alvarez-Arcaya A, Fernández-Díaz E, et al. A prospective study of the management of non-massive pulmonary embolism in the home. Eur J Intern Med 2009;20:598-600

34 Kovacs MJ, Anderson D, Morrow B, et al. Outpatient treatment of pulmonary embolism with dalteparin. Thromb Haemost 2000;83:209-11.

35 Erkens PM, Gandara E, Wells P, et al. Safety of outpatient treatment in acute pulmonary embolism. J Thromb Haemost 2010;8:2412-7.

36 Zondag W, den Exter PL, Crobach MJT, et al. Comparison of two methods for selection of out of hospital treatment in patients with acute pulmonary embolism. Thromb Haemost 2013;109:47-52.

37 Zondag W, Vingerhoets LM, Durian MF, et al. Hestia criteria can safely select patients with pulmonary embolism for outpatient treatment irrespective of right ventricular function. J Thromb Haemost 2013;11:686-92.

38 Squizzato A, Donadini MP, Galli L, et al. Prognostic clinical prediction rules to identify a low-risk pulmonary embolism: a systematic review and meta-analysis. J Thromb Haemost 2012;10:1276-90.

39 Nieto JA, Solano R, Ruiz-Ribó MD, et al. Fatal bleeding in patients receiving anticoagulant therapy for venous thromboembolism: findings from the RIETE registry. J Thromb Haemost 2010;8:1216-22.

40 Ruíz-Giménez N, Suárez C, González R, et al. Predictive variables for major bleeding events in patients presenting with documented acute venous thromboembolism. Findings from the RIETE Registry. Thromb Haemost 2008; 100:26-31
41 Shopp JD, Stewart LK, Emmett TW, et al. Findings From 12-lead Electrocardiography That Predict Circulatory Shock From Pulmonary Embolism: Systematic Review and Meta-analysis. Acad Emerg Med 2015;22:1127-37.

42 Klok FA, Niemann C, Dellas C, et al. Performance of five different bleedingprediction scores in patients with acute pulmonary embolism. J Thromb Thrombolysis 2016:41:312-20.

43 Klok FA, Mos IC, Huisman MV. Brain-type natriuretic peptide levels in the prediction of adverse outcome in patients with pulmonary embolism: a systematic review and meta-analysis. Am J Respir Crit Care Med 2008;178:425-30.

44 Jimenez D, Diaz G, Molina J, et al. Troponin I and risk stratification of patients with acute nonmassive pulmonary embolism. Eur Respir J 2008;31:847-53.

45 Jiménez D, Uresandi F, Otero R, et al. Troponin-based risk stratification of patients with acute nonmassive pulmonary embolism: systematic review and metaanalysis. Chest 2009;136:974-82

46 Moores L, Aujesky D, Jiménez D, et al. Pulmonary Embolism Severity Index and troponin testing for the selection of low-risk patients with acute symptomatic pulmonary embolism. J Thromb Haemost 2010;8:517-22.

47 Lankeit M, Jiménez D, Kostrubiec M, et al. Predictive value of the high-sensitivity troponin T assay and the simplified Pulmonary Embolism Severity Index in hemodynamically stable patients with acute pulmonary embolism: a prospective validation study. Circulation 2011;124:2716-24.

48 Ozsu S, Abul Y, Orem A, et al. Predictive value of troponins and simplified pulmonary embolism severity index in patients with normotensive pulmonary embolism. Multidiscip Respir Med 2013:8:34.

49 Hakemi EU, Alyousef T, Dang G, et al. The prognostic value of undetectable highly sensitive cardiac troponin I in patients with acute pulmonary embolism. Chest 2015;147:685-94.

50 Jiménez D, Escobar C, Martí D, et al. [Prognostic value of transthoracic echocardiography in hemodynamically stable patients with acute symptomatic pulmonary embolism]. Arch Bronconeumol 2007;43:490-4.

51 Coutance G, Cauderlier E, Ehtisham J, et al. The prognostic value of markers of right ventricular dysfunction in pulmonary embolism: a meta-analysis. Critical Care 2011:15:R103.

52 Becattini C, Agnelli G, Germini F, et al. Computed tomography to assess risk of death in acute pulmonary embolism: a meta-analysis. Eur Respir J 2014;43:1678-90.

53 Jiménez D, Lobo JL, Monreal M, et al. Prognostic significance of multidetector CT in normotensive patients with pulmonary embolism: results of the protect study. Thorax 2014;69:109-15.

54 Jiménez D, Aujesky D, Díaz G, et al. Prognostic significance of deep vein thrombosis in patients presenting with acute symptomatic pulmonary embolism. Am J Respir Crit Care Med 2010;181:983-91.

55 Aujesky D, Roy PM, Guy M, et al. Prognostic value of D-dimer in patients with pulmonary embolism. Thromb Haemost 2006:96:478-82.

56 Vanni S, Jimenez D, Nazerian P, et al. Prognostic value of plasma lactate in acute pulmonary embolism: the multicentre Thrombo-Embolism Lactate Outcome study. Eur Heart J 2013:34:758

57 Jiménez D, Kopecna D, Tapson V, et al. Derivation and validation of multimarker prognostication for normotensive patients with acute symptomatic pulmonary embolism. Am J Respir Crit Care Med 2014;189:718-26.

58 Vuilleumier N, Limacher A, Méan M, et al. Cardiac biomarkers and clinical scores for risk stratification in elderly patients with non-high-risk pulmonary embolism. J Intern Med 2015;277:707-16.

59 Hogg K, Dawson D, Mackway-Jones K. Outpatient diagnosis of pulmonary embolism: the MIOPED (Manchester Investigation Of Pulmonary Embolism Diagnosis) study. Emerg Med J 2006;23:123-7.

60 McDonald AH, Murphy R. A pilot audit of a protocol for ambulatory investigation of predicted low-risk patients with possible pulmonary embolism. J $R$ Coll Physicians Edinb 2011;41:196-201

61 Gómez-Outes A, Terleira-Fernández Al, Lecumberri R, et al. Direct oral anticoagulants in the treatment of acute venous thromboembolism: A systematic review and metaanalysis. Thromb Res 2014;134:774-82.

62 Schulman S, Kearon C, Kakkar AK, et al. Dabigatran versus warfarin in the treatment of acute venous thromboembolism. N Engl J Med Overseas Ed 2009:361:2342-52.

63 Investigators E-P, Buller HR, Prins MH, et al. Oral rivaroxaban for the treatment of symptomatic pulmonary embolism. N Eng/ J Med Overseas Ed 2012;366:1287-97.

64 Agnelli G, Buller HR, Cohen A, et al. Oral apixaban for the treatment of acute venous thromboembolism. N Engl J Med Overseas Ed 2013;369:799-808.

65 Büller HR, Décousus H, Grosso MA, et al. Edoxaban versus warfarin for the treatment of symptomatic venous thromboembolism. N Eng/ J Med 2013;369:1406-15.

66 Robertson L, Kesteven P, McCaslin JE. Oral direct thrombin inhibitors or oral factor Xa inhibitors for the treatment of pulmonary embolism. Cochrane Database Syst Rev 2015:4:CD010957.

67 Prins $\mathrm{MH}$, Lensing AWA, Bauersachs $\mathrm{R}$, et al. Oral rivaroxaban versus standard therapy for the treatment of symptomatic venous thromboembolism: a pooled analysis of the EINSTEIN-DVT and PE randomized studies. Thromb J 2013;11:21. 
68 Sardar P, Chatterjee S, Lavie CJ, et al. Risk of major bleeding in different indications for new oral anticoagulants: Insights from a meta-analysis of approved dosages from 50 randomized trials. Int J Cardio/ 2015;179:279-87.

69 van Es N, Coppens M, Schulman S, et al. Direct oral anticoagulants compared with vitamin $\mathrm{K}$ antagonists for acute venous thromboembolism: evidence from phase 3 trials. Blood 2014;124:1968-75.

70 Prandoni P, Prins MH, Cohen AT, et al. Use of prestudy heparin did not influence the efficacy and safety of rivaroxaban in patients treated for symptomatic venous thromboem-bolism in the EINSTEIN DVT and EINSTEIN PE studies. Academic Emergency Medicine 2015;22:142-9.

71 Beam DM, Kahler ZP, Kline JA. Immediate discharge and home treatment with rivaroxaban of low-risk venous thromboembolism diagnosed in Two U.S. emergency departments: a one-year preplanned analysis. Acad Emerg Med 2015;22:788-95.

72 Kline J, Beam D, Kahler Z. Outpatient treatment of low-risk venous thromboembolism with monotherapy oral anticoagulation: patient quality of life outcomes and clinician acceptance. Patient Prefer Adherence 2016;10:561-9.

73 van Bellen B, Bamber L, Correa de Carvalho F, et al. Reduction in the length of stay with rivaroxaban as a single-drug regimen for the treatment of deep vein thrombosis and pulmonary embolism. Curr Med Res Opin 2014;30:829-37.

74 Bookhart BK, Haskell L, Bamber L, et al. Length of stay and economic consequences with rivaroxaban vs enoxaparin/vitamin $\mathrm{K}$ antagonist in patients with DVT and PE: findings from the North American EINSTEIN clinical trial program. J Med Econ 2014;17:691-5.

75 Matsuo H, Prins M, Lensing AWA, et al. Shortened length of hospital stay with rivaroxaban in patients with symptomatic venous thromboembolism in Japan: the J-EINSTEIN pulmonary embolism and deep vein thrombosis program. Curr Med Res Opin 2015;31:1057-61.

76 Roberts KM, Knight TB, Padilla-Tolentino E, et al. Length of stay comparison between rivaroxaban and warfarin in the treatment of pulmonary embolism: results from a real-world observational cohort study. Thrombosis 2015;2015:414523-

77 Masotti L, Vannucchi V, Poggi M, et al. Trends in length of hospital stay in acute pulmonary embolism over the years. What is changing in the era of direct oral anticoagulants? J Cardiovasc Med 2017;18:556-559.

78 Moores L, Zamarro C, Gómez V, et al. Changes in PESI scores predict mortality in intermediate-risk patients with acute pulmonary embolism. Eur Respir J 2013;41:354-9.

79 Medicine RCoE. Clinical effectiveness committee standard: consultant sign-off, 2010.

80 Misky GJ, Carlson T, Thompson E, et al. Implementation of an acute venous thromboembolism clinical pathway reduces healthcare utilization and mitigates health disparities. J Hosp Med 2014;9:430-5.

81 Lim AY, Parr DG, Stableforth DE, et al. Early discharge and home supervision of patients with pulmonary embolism treated with low-molecular weight heparin. Eur J Intern Med 2003;14:89-93.

82 Watson H, Davidson S, Keeling D, et al. Guidelines on the diagnosis and management of heparin-induced thrombocytopenia: second edition. Br J Haematol 2012:129:n/a-40.

83 Cardiology ESo. Diagnosis and Management of Acute Pulmonary Embolism. Eur Heart J 2014. doi: 10.1093/eurheartj/ehu283 [Epub ahead of print].

84 Piccioli A, Lensing AW, Prins MH, et al. Extensive screening for occult malignant disease in idiopathic venous thromboembolism: a prospective randomized clinical trial. J Thromb Haemost 2004;2:884-9.

85 Van Doormaal FF, Terpstra W, Van Der Griend R, et al. Is extensive screening for cancer in idiopathic venous thromboembolism warranted? J Thromb Haemost 2011;9:79-84.

86 Carrier M, Lazo-Langner A, Shivakumar S, et al. Screening for occult cancer in unprovoked venous thromboembolism. N Engl J Med Overseas Ed 2015:373:697-704.

87 Knight M, Nair M, Tuffnell D, et al. Saving Lives, Improving Mothers' Care. Surveillance of maternal deaths in the UK 2012-14 and lessons learned to inform maternity care from the UK and Ireland Confidential Enquiries into Maternal Deaths and Morbidity 2009-14. Oxford: Maternal, Newborn and Infant Clinical Outcome Review Programme, National Perinatal Epidemiology Unit, University of Oxford, 2016.

88 Knight M. UKOSS. Antenatal pulmonary embolism: risk factors, management and outcomes. BJOG 2008:115:453-61.
89 Sultan AA, West J, Tata LJ, et al. Risk of first venous thromboembolism in and around pregnancy: a population-based cohort study. Br J Haematol 2012;156:366-73.

90 Thrombosis and embolism during pregnancy and the puerperium, reducing the risk (Green-top Guideline No. 37a). 2015 13/4/2015. 2015 https://www.rcog.org.uk/en/ guidelines-research-services/guidelines/gtg37a/

91 Thrombosis and embolism during pregnancy and the puerperium, the acute management of (Green-top Guideline No. 37b). 2015 13/4/2015. 2015 https:// www.rcog.org.uk/en/guidelines-research-services/guidelines/gtg37b/

92 Joung S, Robinson B. Venous thromboembolism in cancer patients in Christchurch, 1995-1999. The New Zealand medical journal 2002;115:257-60.

93 den Exter PL, Hooijer J, Dekkers OM, et al. Risk of recurrent venous thromboembolism and mortality in patients with cancer incidentally diagnosed with pulmonary embolism: a comparison with symptomatic patients. J Clin Oncol 2011;29:2405-9.

94 Lee AYY, Levine MN. Venous thromboembolism and cancer: risks and outcomes. Circulation 2003:107:17|--21.

95 Heit JA, Mohr DN, Silverstein MD, et al. Predictors of recurrence after deep vein thrombosis and pulmonary embolism: a population-based cohort study. Arch Intern Med 2000;160:761-8.

96 Hutten BA, Prins MH, Gent M, et al. Incidence of recurrent thromboembolic and bleeding complications among patients with venous thromboembolism in relation to both malignancy and achieved international normalized ratio: a retrospective analysis. J Clin Oncol 2000;18:3078-83.

97 den Exter PL, Gómez V Jiménez D, et al. A clinical prognostic model for the identification of low-risk patients with acute symptomatic pulmonary embolism and active cancer. Chest 2013;143:138-45

98 Kline JAK, C.:Beam D. Hestia criteria with the POMPE-C tool identifies patients with cancer and pulmonary embolism at very low risk for short-term complications. Journal of Thrombosis and Haemostasis 2013:11:864.

99 Kline JA, Roy P-M, Than MP, et al. Derivation and validation of a multivariate model to predict mortality from pulmonary embolism with cancer: The POMPE-C tool. Thromb Res 2012:129:e194-e199.

100 Font C, Carmona-Bayonas A, Fernández-Martinez A, et al. Outpatient management of pulmonary embolism in cancer: data on a prospective cohort of 138 consecutive patients. J Nat/ Compr Canc Netw 2014;12:365-73.

101 Siragusa S, Anastasio R, Giarratano A, et al. Managing cancer patients with acute venous thromboembolism: exploring safe alternatives to hospitalisation. Exp Oncol 2004:26:192-5.

102 Siragusa S, Arcara C, Malato A, et al. Home therapy for deep vein thrombosis and pulmonary embolism in cancer patients. Ann Oncol 2005;16(suppl_4):iv136 -iv139.

103 Ageno W, Grimwood R, Limbiati S, et al. Home-treatment of deep vein thrombosis in patients with cancer. Haematologica 2005;90:220-4.

104 Kovacs MJ, Hawel JD, Rekman JF, et al. Ambulatory management of pulmonary embolism: a pragmatic evaluation. J Thromb Haemost 2010;8:2406-11.

105 Syed FF, Beeching NJ. Lower-limb deep-vein thrombosis in a general hospital: risk factors, outcomes and the contribution of intravenous drug use. QJM 2005;98:139-45.

106 Cooke VA, Fletcher AK. Deep vein thrombosis among injecting drug users in Sheffield. Emerg Med J 2006;23:777-9.

107 McColl MD, Tait RC, Greer IA, et al. Injecting drug use is a risk factor for deep vein thrombosis in women in Glasgow. Br J Haematol 2001;112:641-3.

108 Labropoulos N, Giannoukas AD, Leon M, et al. Complications after intravenous drug abuse and the diagnostic value of color flow duplex scanning. Journal of Vascular Technology 1996;20:27-8

109 Mackenzie AR, Laing RB, Douglas JG, et al. High prevalence of iliofemoral venous thrombosis with severe groin infection among injecting drug users in North East Scotland: successful use of low molecular weight heparin with antibiotics. Postgrad Med J 2000;76:561-5

110 Anderson AM, Chane T, Patel M, et al. Warfarin therapy in the HIV medical home model: low rates of therapeutic anticoagulation despite adherence and differences in dosing based on specific antiretrovirals. AIDS Patient Care STDS 2012:26:454-62. 


\section{APPENDIX 1: GUIDELINE GROUP MEMBERS}

\section{British Thoracic Society Outpatient Management of Pulmonary Embolism}

\section{Guideline Development Group}

Dr Luke Howard, Chair

Dr Steve Barden

Dr Robin Condliffe

Dr Vincent Connolly, representing the Society for Acute Medicine

Dr Chris Davies, representing the Royal College of Physicians

Dr James Donaldson

Mr Bernard Everett, lay/patient representative

Dr Catherine Free

Dr Daniel Horner, representing the College of Emergency Medicine

Dr Laura Hunter, representing the College of Emergency Medicine

Mr Jasvinder Kaler

Dr Cathy Nelson-Piercy

Dr Emma O’Dowd

Dr Raj Patel

Ms Wendy Preston, representing the Association of Respiratory Nurse Specialists

Dr Karen Sheares

Dr Campbell Tait, representing the British Committee for Standards in Haematology

Dr Rodney Hughes, member from February to December 2014

Dr Matthew Fay, member from February 2014 to January 2015

\section{Competing interests}

RC received funding from BTG, Actelion,MSD; BE received funding from Astra Zeneca, Diageo; DH recived finding from Creavotech; LH received funding from GSK, Endotronix, Actelion, Bayer, MSD, BTG, BMS; RP received funding from Bayer, Daiichi Sankyo; KS received funding from Actelion; CT received funding from Bayer, Daiichi Sankyo, Novo Nordisk; CNP received funding from UCB, Alliance; WP received funding from ARNS, AZ, BI

No interests to declare: SB, CD, JD, CF, LH, EO’D, JK 


\section{BTS guideline}

\section{APPENDIX 2: SUMMARY OF RISK SCORES}

\begin{tabular}{|c|c|c|c|c|c|c|c|c|}
\hline 商 & 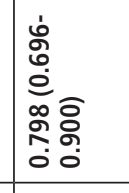 & $\frac{\pi}{2}$ & $\mid$ & & $\begin{array}{l}\frac{5}{2} \\
\frac{\cos }{2} \\
\frac{2}{2}\end{array}$ & 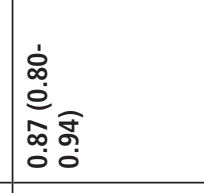 & 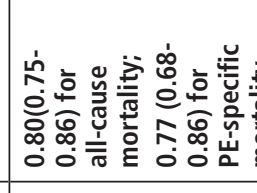 & 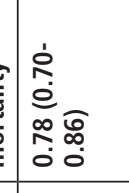 \\
\hline & 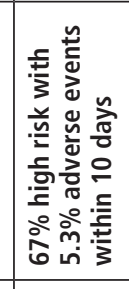 & 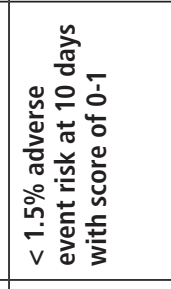 & 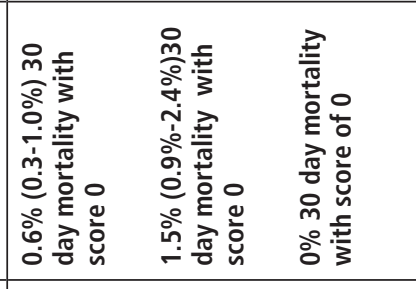 & & 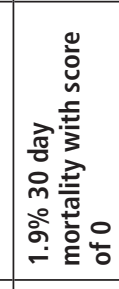 & 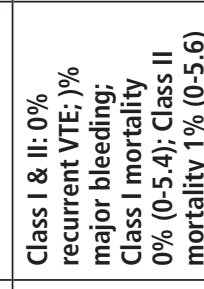 & 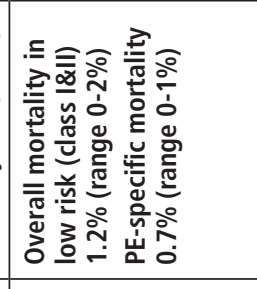 & 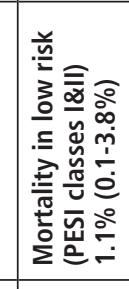 \\
\hline & 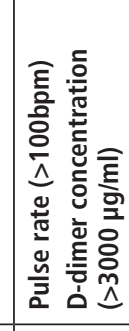 & 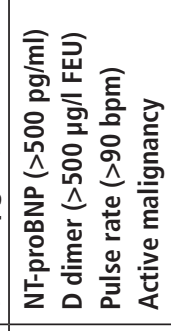 & 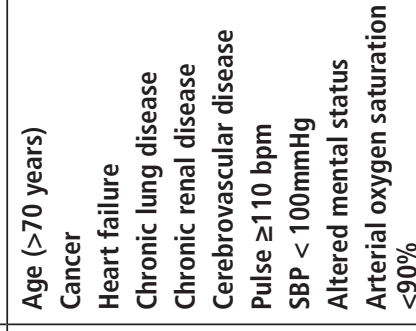 & & 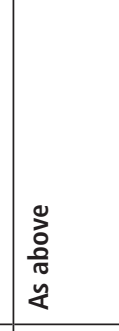 & 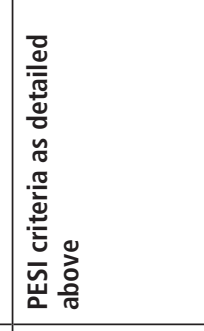 & 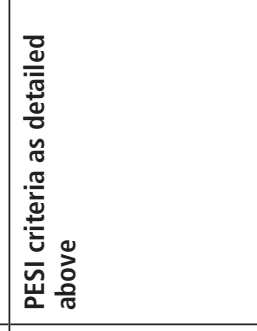 & 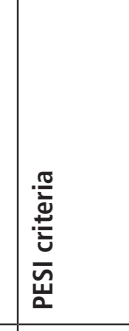 \\
\hline 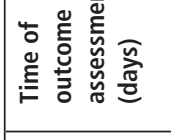 & $\begin{array}{l}0 \\
2 \\
\infty \\
0 \\
0\end{array}$ & $\because$ & $\stackrel{\circ}{m}$ & $\stackrel{\circ}{m}$ & $\stackrel{\circ}{m}$ & 8 & 8 & 8 \\
\hline 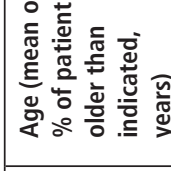 & 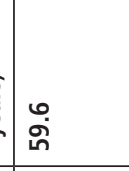 & : & 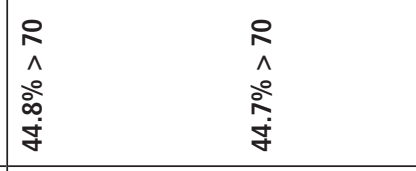 & 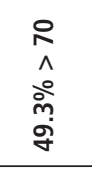 & m & 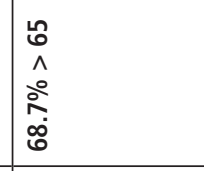 & 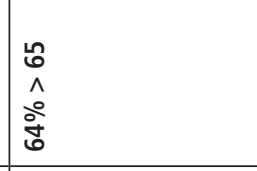 & It \\
\hline 离 & 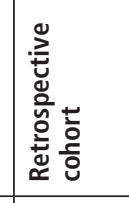 & 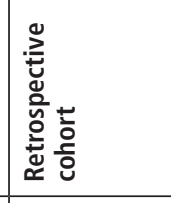 & 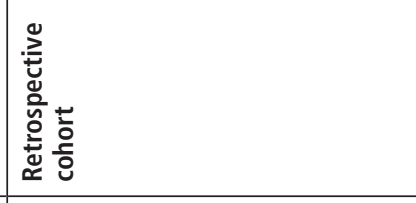 & & 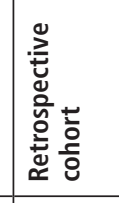 & 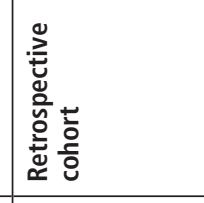 & 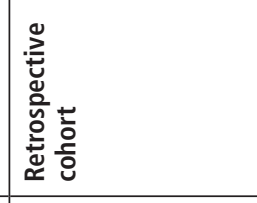 & 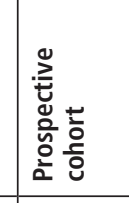 \\
\hline 意䓌 & 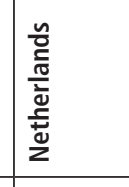 & 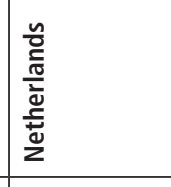 & $\stackrel{\mathbb{J}}{\mathrm{s}}$ & 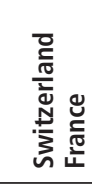 & 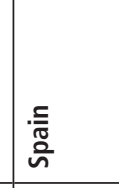 & 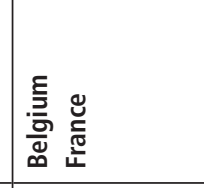 & 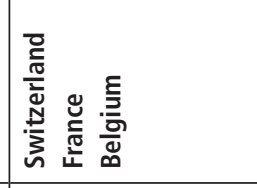 & 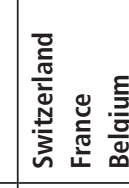 \\
\hline 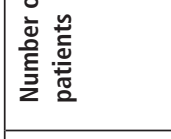 & f & 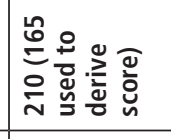 & 㟥 & $\bar{i}$ & $\begin{array}{c}\infty \\
\stackrel{\infty}{\circ}\end{array}$ & $\stackrel{్}{0}$ & ळ & 隹 \\
\hline 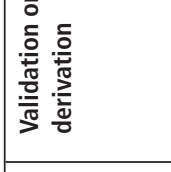 & 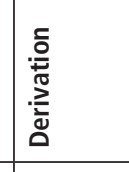 & 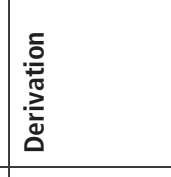 & 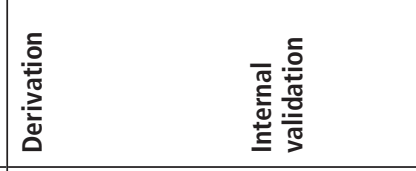 & 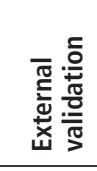 & 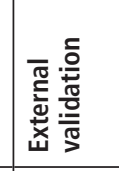 & 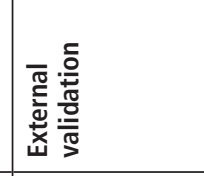 & 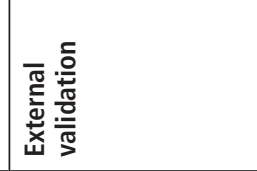 & 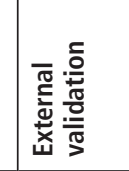 \\
\hline 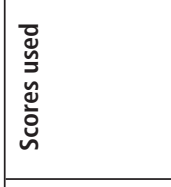 & 咅 & 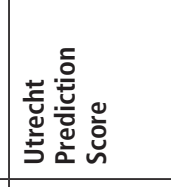 & 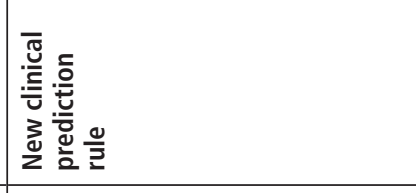 & & 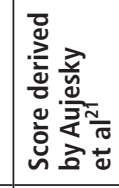 & $\overline{\bar{g}}$ & 台 & 产 \\
\hline 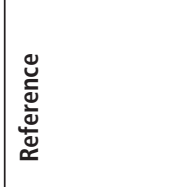 & 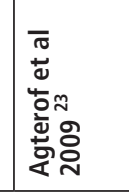 & 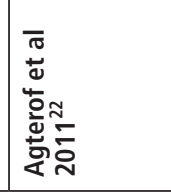 & 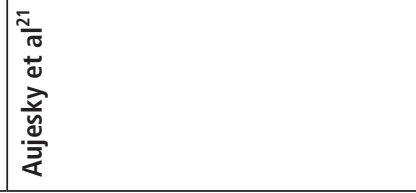 & & 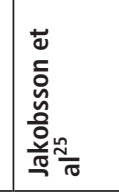 & 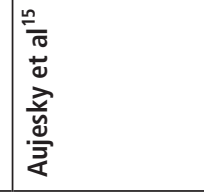 & 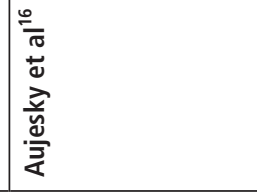 & 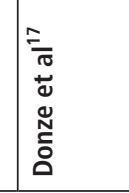 \\
\hline
\end{tabular}




\begin{tabular}{|c|c|c|c|c|c|c|c|}
\hline 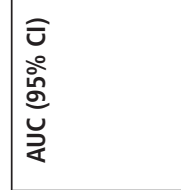 & 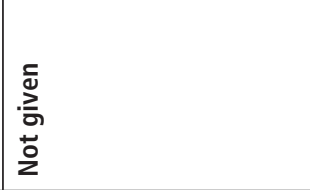 & & 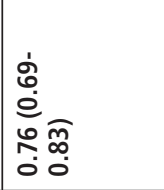 & 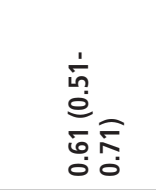 & 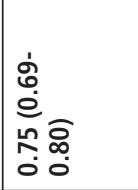 & & 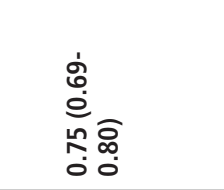 \\
\hline 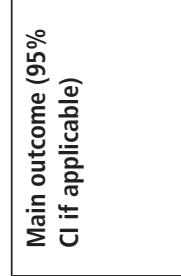 & 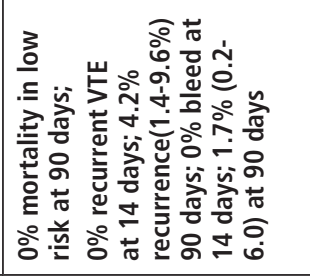 & 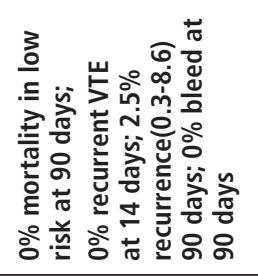 & 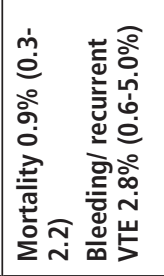 & 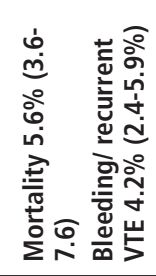 & 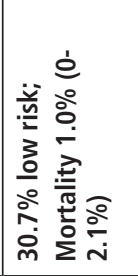 & 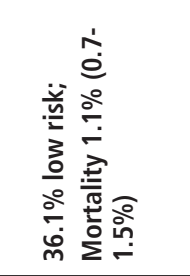 & 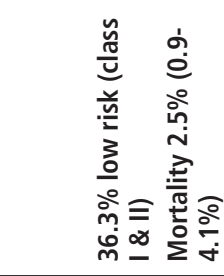 \\
\hline . & 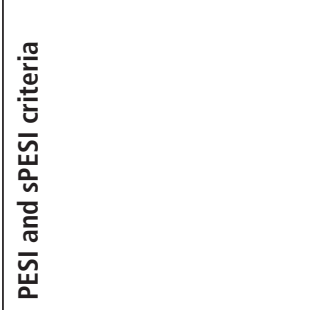 & & 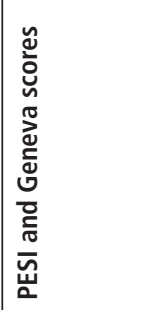 & & 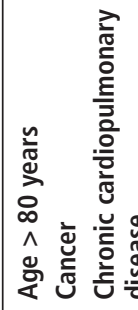 & 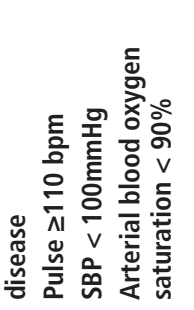 & 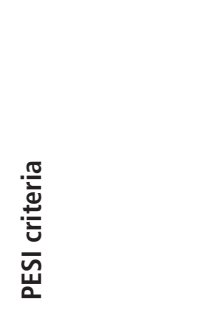 \\
\hline 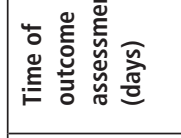 & \pm 용 & & $\stackrel{m}{m}$ & & m & m & m \\
\hline 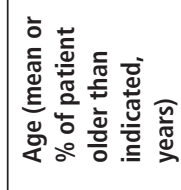 & 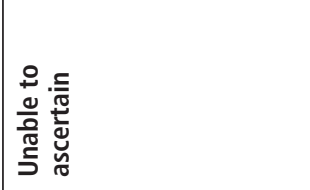 & & $\widehat{\jmath}$ & & 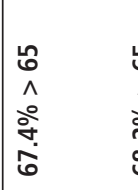 & 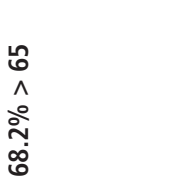 & $\begin{array}{l}\stackrel{0}{0} \\
\stackrel{\circ}{o} \\
\stackrel{0}{0}\end{array}$ \\
\hline 莺 & 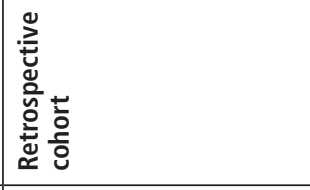 & & 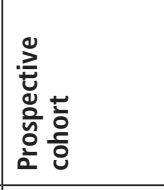 & & 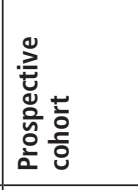 & 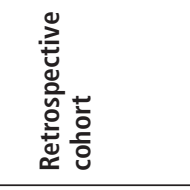 & 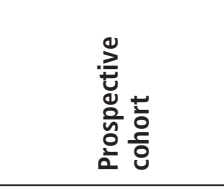 \\
\hline 鿖喜 & 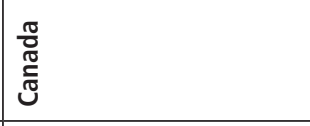 & & 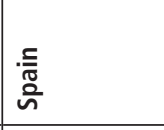 & & \begin{tabular}{|l} 
竞 \\
ñ
\end{tabular} & 崖 & 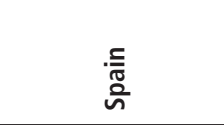 \\
\hline 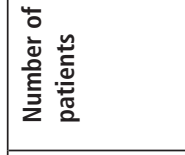 & I & & 煦 & & 号 & $\stackrel{\circ}{2}$ & ถั \\
\hline 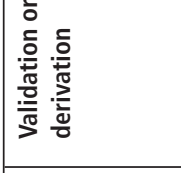 & 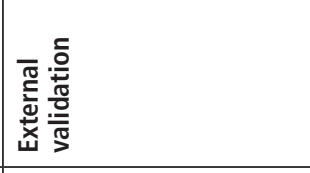 & & 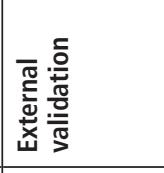 & & 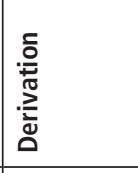 & 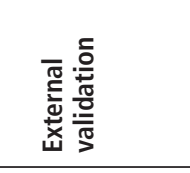 & 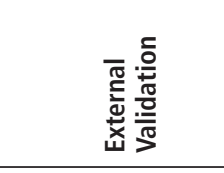 \\
\hline 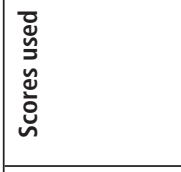 & 噐 & 㐔 & $\overline{\underline{B}}$ & 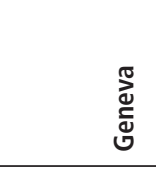 & $\begin{array}{l}\bar{y} \\
\underline{u} \\
\underline{u}\end{array}$ & & 可 \\
\hline 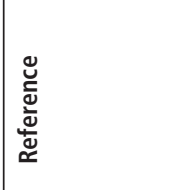 & 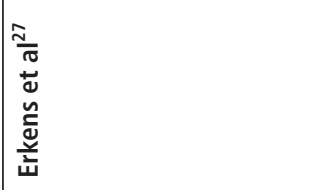 & & 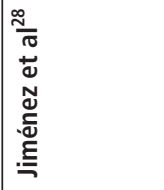 & & 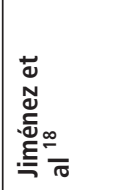 & & \\
\hline
\end{tabular}




\begin{tabular}{|c|c|c|c|c|c|c|c|c|c|c|c|}
\hline 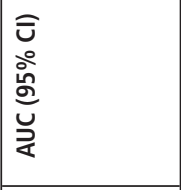 & 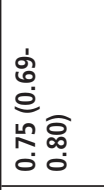 & 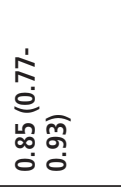 & 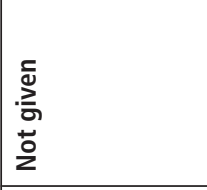 & 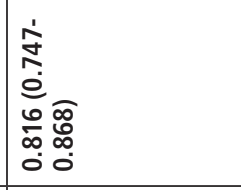 & 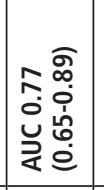 & \begin{tabular}{|l} 
\\
$\frac{\pi}{5}$ \\
$\frac{\pi}{50}$ \\
20 \\
2 \\
\end{tabular} & & 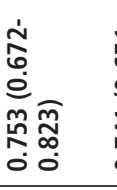 & 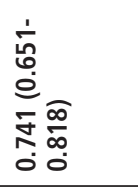 & & \\
\hline 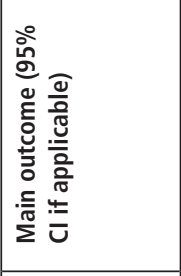 & 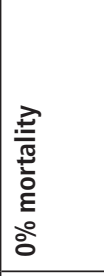 & & 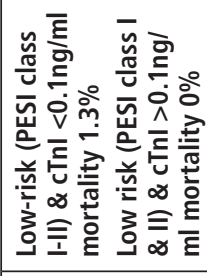 & 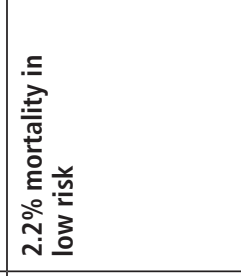 & 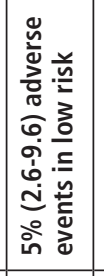 & 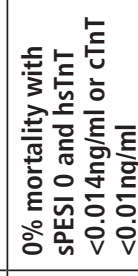 & 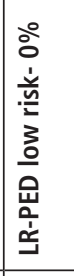 & 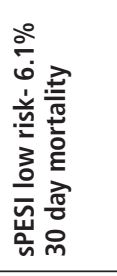 & 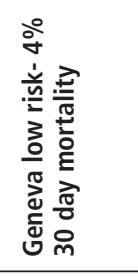 & & \\
\hline 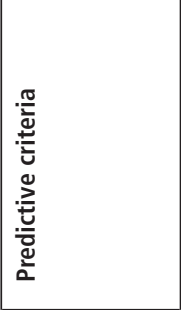 & 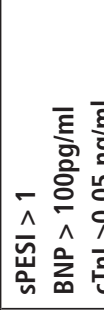 & 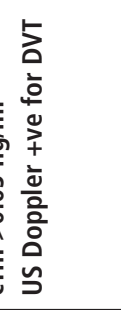 & 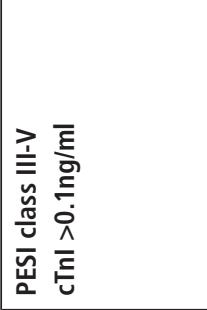 & 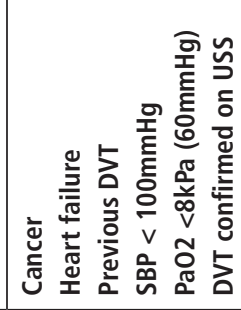 & 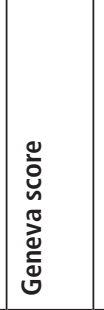 & 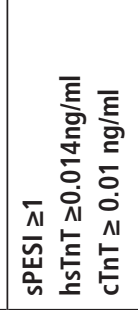 & \multicolumn{5}{|c|}{ 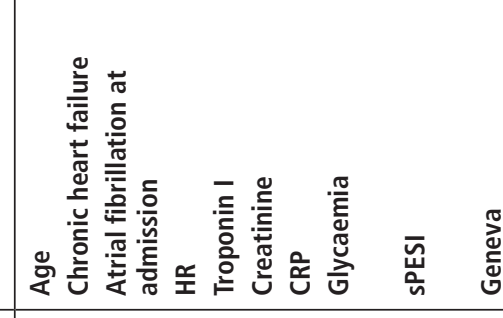 } \\
\hline 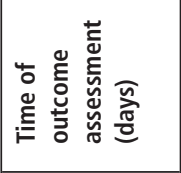 & m & & m & 8 & 8 & 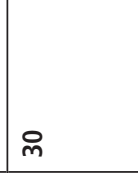 & & & & & \\
\hline 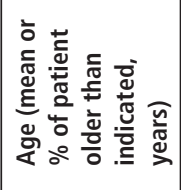 & 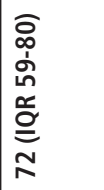 & 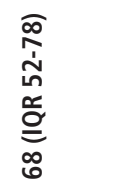 & 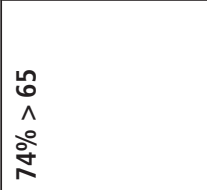 & $\mid \begin{array}{l}n \\
6\end{array}$ & 苗 & 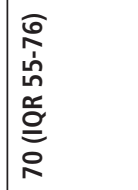 & 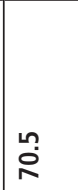 & & & & \\
\hline 咅 & 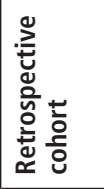 & 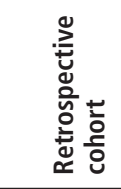 & 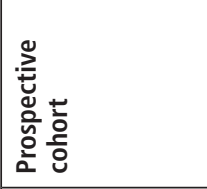 & 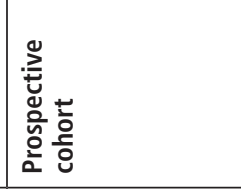 & 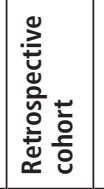 & 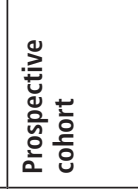 & 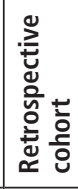 & & & & \\
\hline 意眷 & 爰 & & $\mid \begin{array}{l}\frac{c}{1} \\
\text { 崽 }\end{array}$ & 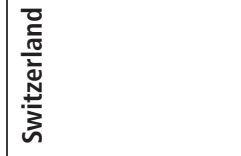 & 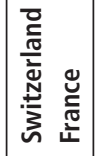 & 离 & \begin{tabular}{|l} 
爱 \\
产
\end{tabular} & & & & \\
\hline 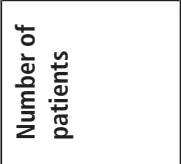 & 迹 & กิ & 占 & 足 & $\tilde{\approx}$ & $\bar{\beth}$ & $\exists$ & & & & \\
\hline 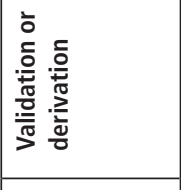 & 旁 & 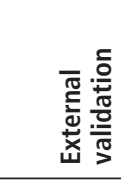 & 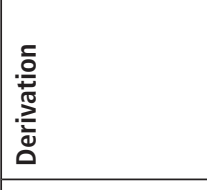 & 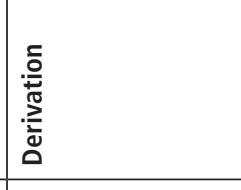 & 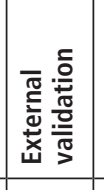 & 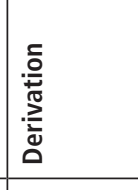 & 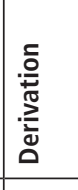 & 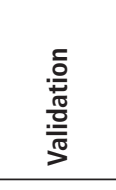 & & 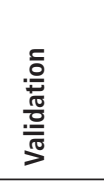 & \\
\hline 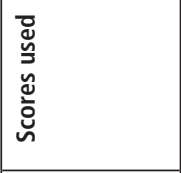 & 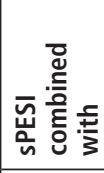 & & 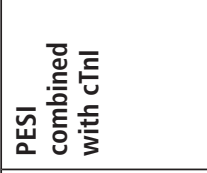 & 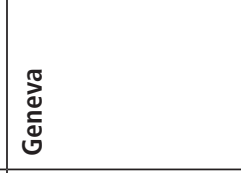 & 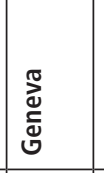 & 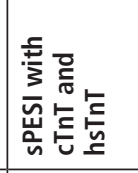 & 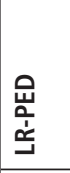 & 产 & & 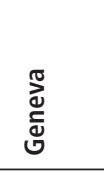 & \\
\hline 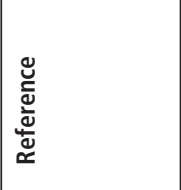 & 芯 & & 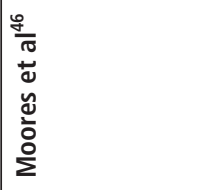 & 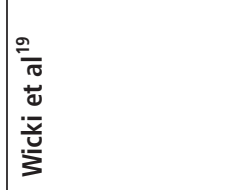 & 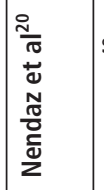 & 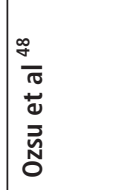 & 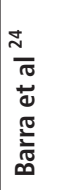 & & & & \\
\hline
\end{tabular}




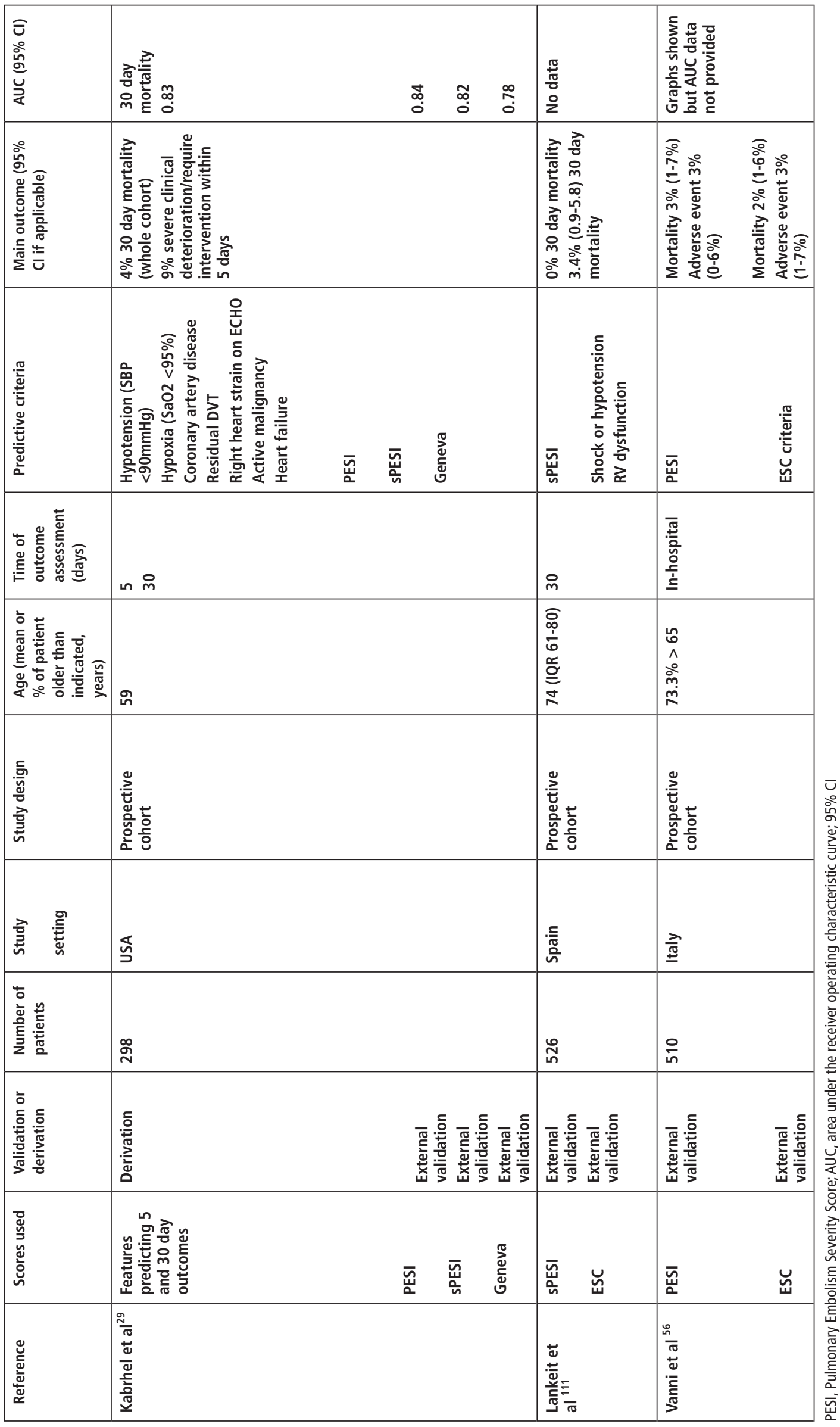

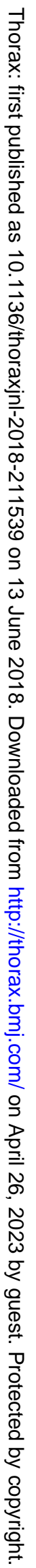




\section{BTS guideline}

\section{APPENDIX 3: SUMMARY OF VALIDATED SCORES}

\begin{tabular}{|c|c|c|c|c|c|c|c|c|c|}
\hline 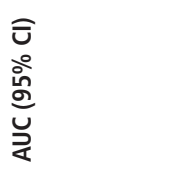 & $\underline{\pi}$ & 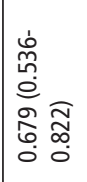 & 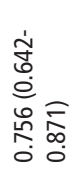 & $\begin{array}{l}\frac{\pi}{0} \\
\frac{50}{0} \\
2 \\
2\end{array}$ & & 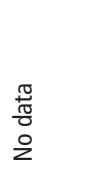 & & 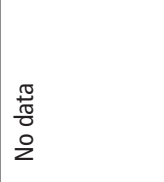 & \\
\hline 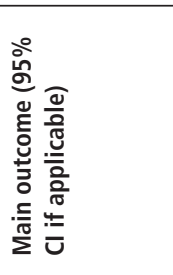 & 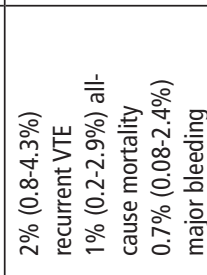 & 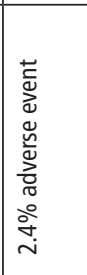 & 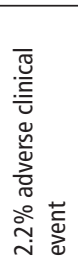 & 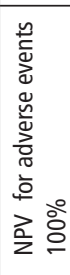 & 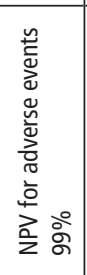 & 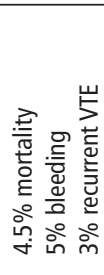 & 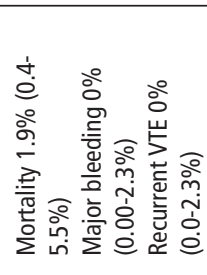 & 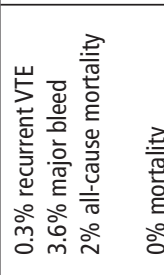 & 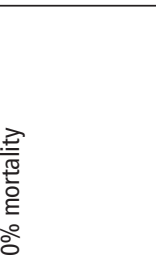 \\
\hline 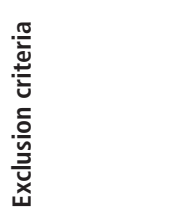 & 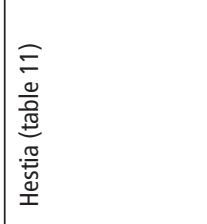 & 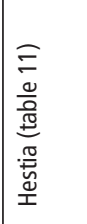 & 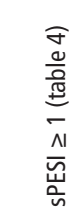 & 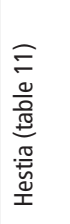 & 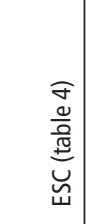 & 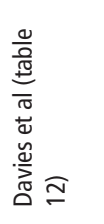 & & 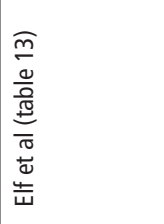 & 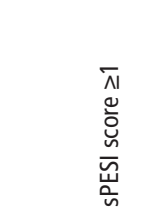 \\
\hline 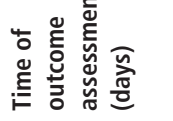 & 8 & m & & 8 & & 8 & 8 & 8 & \\
\hline 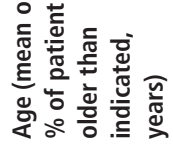 & 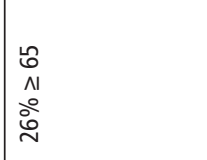 & $\stackrel{\infty}{\circ}$ & & $\stackrel{\infty}{\text { }}$ & & $\frac{\pi}{z}$ & $\infty_{\Perp}^{\infty}$ & ๑ & \\
\hline 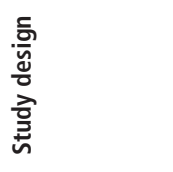 & 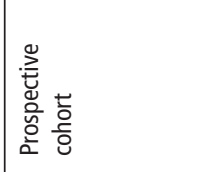 & 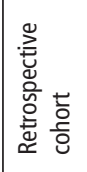 & & 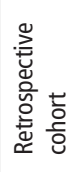 & & 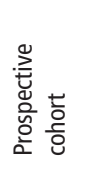 & & 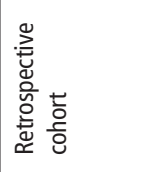 & \\
\hline 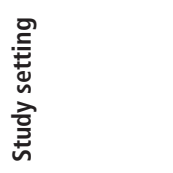 & 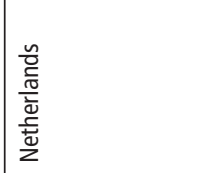 & 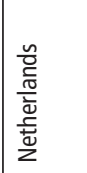 & & 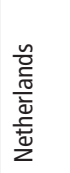 & & 兰 & & 离 & \\
\hline 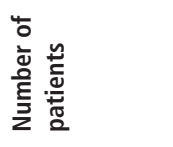 & వે & ‡ & 囟 & $\stackrel{\stackrel{n}{\sim}}{\sim}$ & $\stackrel{\circ}{\circ}$ & $\stackrel{\sim}{\sim}$ & 5 & $\check{\infty}_{\infty}$ & 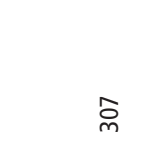 \\
\hline 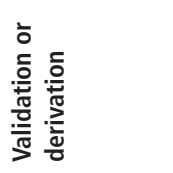 & 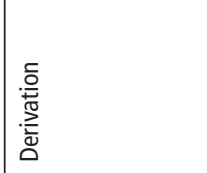 & 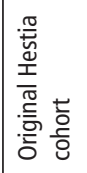 & 要 & 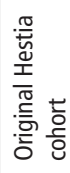 & 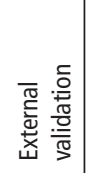 & 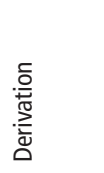 & 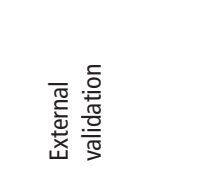 & 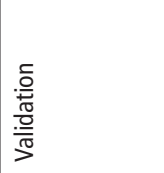 & 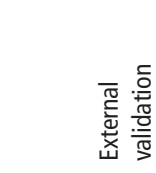 \\
\hline 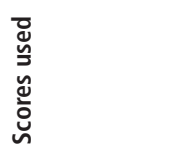 & 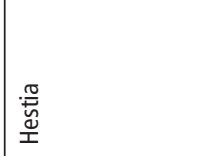 & 噫 & $\overline{\underline{\underline{w}}}$ & 爰 & $\breve{u}$ & 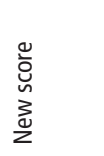 & & 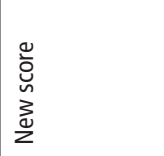 & 产 \\
\hline 营 & 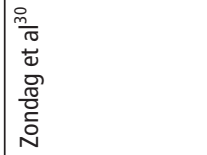 & 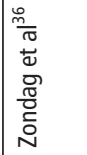 & & 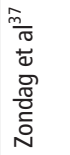 & & T) & & 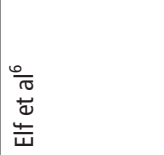 & \\
\hline
\end{tabular}




\begin{tabular}{|c|c|c|c|c|c|}
\hline 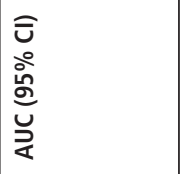 & & & $\begin{array}{l}\frac{\pi}{0} \\
\frac{\pi}{8} \\
0 \\
2\end{array}$ & $\Sigma$ & $\Sigma$ \\
\hline 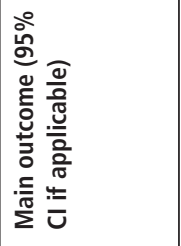 & 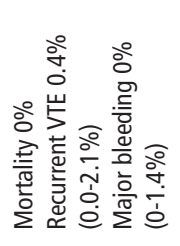 & 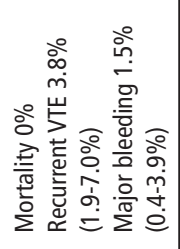 & 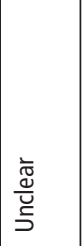 & 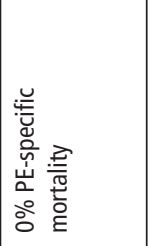 & 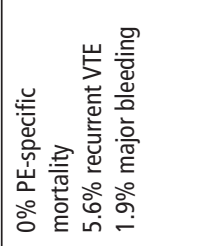 \\
\hline 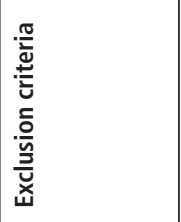 & 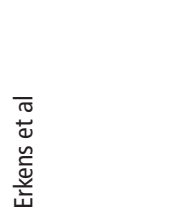 & & 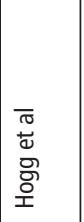 & 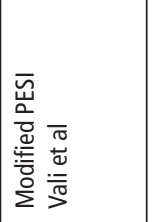 & \begin{tabular}{|l}
$\bar{\pi}$ \\
$\bar{\sigma}$ \\
$\tilde{y}$ \\
$\underline{\tilde{g}}$ \\
$\underline{\partial}$
\end{tabular} \\
\hline 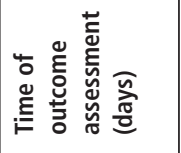 & $\Xi$ & 8 & 8 & ৪ & ৪ \\
\hline 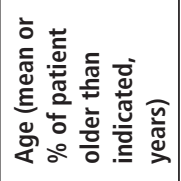 & 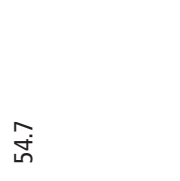 & & $\stackrel{m}{\infty} \underset{m}{m}$ & 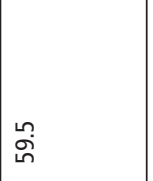 & 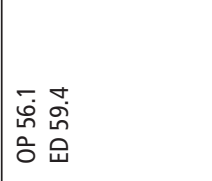 \\
\hline 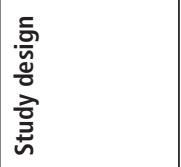 & 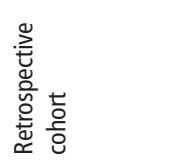 & & 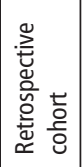 & 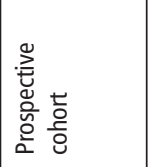 & 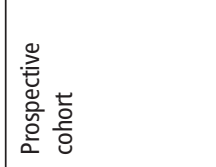 \\
\hline 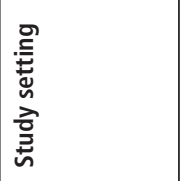 & 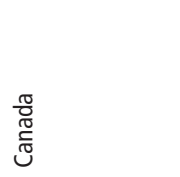 & & 兰 & 兰 & 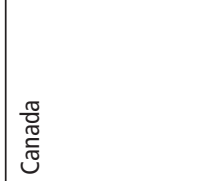 \\
\hline 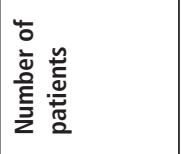 & $\stackrel{m}{f}$ & & 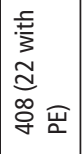 & ஃ & 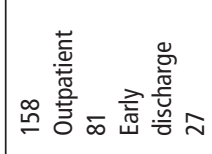 \\
\hline 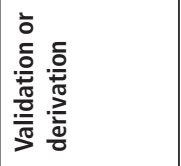 & 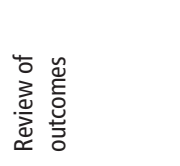 & & 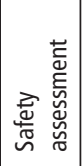 & 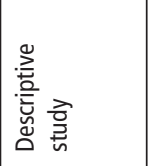 & 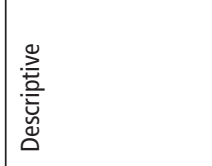 \\
\hline 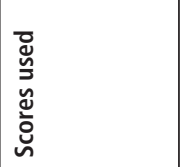 & 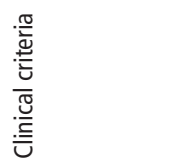 & & 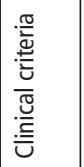 & 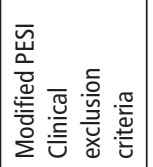 & 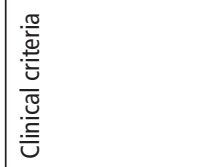 \\
\hline 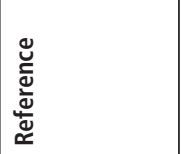 & 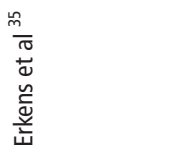 & & 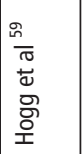 & 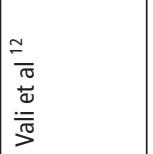 & 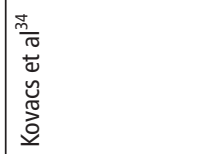 \\
\hline
\end{tabular}

